\title{
The globular cluster system of NGC 1316
}

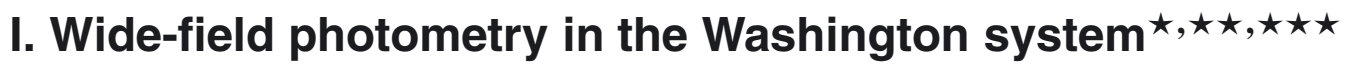

\author{
T. Richtler ${ }^{1}$, L. P. Bassino ${ }^{2}$, B. Dirsch ${ }^{3}$, and B. Kumar ${ }^{4}$ \\ 1 Departamento de Astronomía, Universidad de Concepción, Casilla 160-C, Concepción, Chile \\ e-mail: tom@astro-udec.cl \\ 2 Facultad de Ciencias Astronómicas y Geofísicas de la Universidad Nacional de La Plata; Consejo Nacional de Investigaciones \\ Científicas y Técnicas; and Instituto de Astrofísica de La Plata (CCT La Plata-CONICET-UNLP), Argentina \\ 3 Friedrich-Ebert-Gymnasium, Ollenhauerstrasse 5, 53113 Bonn, Germany \\ 4 Aryabhatta Research Institute of Observational Sciences, Manora Peak, 263129 Nainital, India
}

Received 5 December 2011 / Accepted 13 March 2012

\section{ABSTRACT}

\begin{abstract}
Context. NGC 1316 (Fornax A) is a prominent merger remnant in the outskirts of the Fornax cluster. The bulge stellar population of NGC 1316 has a strong intermediate-age component. Studies of its globular cluster system may help to further refine its probably complex star formation history.

Aims. The cluster system has not yet been studied in its entirety. We therefore present a wide-field study of the globular cluster system of NGC 1316, investigating its properties in relation to the global morphology of NGC 1316.

Methods. We used the MOSAIC II camera at the 4-m Blanco telescope at CTIO in the filters Washington C and Harris R. We identified globular cluster candidates and studied their color distribution and the structural properties of the system. In an appendix, we also remark on the morphology, present color maps, and present new models for the brightness and color profiles of the galaxy.

Results. The cluster system is well confined to the optically visible outer contours of NGC 1316. There are about 640 cluster candidates down to $R=24 \mathrm{mag}$. The color distribution of the entire sample is unimodal, but the color distribution of bright subsamples in the bulge shows two peaks that, compared with theoretical Washington colors with solar metallicity, correspond to ages of about $2 \mathrm{Gyr}$ and $0.8 \mathrm{Gyr}$, respectively. We also find a significant population of clusters in the color range $0.8<C-R<1.1$, which must be populated by clusters younger than $0.8 \mathrm{Gyr}$, unless they are very metal-poor. The color interval $1.3<C-R<1.6$ hosts the bulk of intermediate-age clusters, which show a surface density profile with a sharp decline at about $4^{\prime}$. The outer cluster population shows an unimodal color distribution with a peak at $C-R=1.1$, indicating a higher contribution of old, metal-poor clusters. However, their luminosity function does not show the expected turn-over, so the fraction of younger clusters is still significant. We find a pronounced concentration of blue cluster candidates in the area of Schweizer's L1-structure.

Conclusions. Cluster formation in NGC 1316 has continued after an initial burst that is presumably related to the main merger. A toy model with two bursts of ages $2 \mathrm{Gyr}$ and $0.8 \mathrm{Gyr}$ is consistent with photometric properties and dynamical $M / L$-values. In this model, the older, metal-rich pre-merger population has an age of $7 \mathrm{Gyr}$, contributes $90 \%$ of the bulge mass and $70 \%$ of the luminosity. Its properties are consistent with spiral galaxies, where star-bursts were triggered by major/minor mergers and/or close encounters.
\end{abstract}

Key words. galaxies: individual: NGC 1316 - galaxies: star clusters: general - galaxies: peculiar

\section{Introduction}

The study of globular clusters systems (GCSs) is motivated by the hope of finding clues to the formation and history of their host galaxies (for reviews see Brodie \& Strader 2006; Harris 2010). Globular cluster (GC) formation occurs in a large variety of star-forming environments and at all epochs.

The formation of massive clusters is apparently favored in star bursts because they occur in merger events (e.g. Whitmore \& Schweizer 1995; de Grijs et al. 2003b), but one finds GCs also in star-forming disks of normal spiral galaxies where enhanced star formation rates again seem to be related to an enhanced

\footnotetext{
* Based on observations obtained at the Cerro Tololo Inter-American Observatory, Chile.

$\star \star$ Appendix is available in electronic form at

http://www . aanda.org

$\star \star \star$ The photometric data are only available at the CDS via anonymous ftp to cdsarc.u-strasbg.fr (130.79.128.5) or via

http://cdsarc.u-strasbg.fr/viz-bin/qcat?]/A+A/543/A131
}

efficiency of massive cluster formation (Larsen \& Richtler 1999, 2000). Against traditional wisdom, intermediate-age globular clusters exist even in the Milky Way (Davies et al. 2011).

The old globular cluster systems of elliptical galaxies exhibit characteristic properties. A striking pattern is the colorbimodality, featuring a blue (bona fide metal-poor) peak and a red (bona fide metal-rich) peak (e.g. Larsen et al. 2001; Kundu \& Whitmore 2001).

Because early merger events are believed to be the driver for the formation of elliptical galaxies, the properties of GCSs of known merger remnants may provide more insight into the formation mechanisms.

The nearest merger remnant is NGC 5128 (Cen A) at a distance of $3.8 \mathrm{Mpc}$. Its stellar population is best described by the bulk of stars (about $80 \%$ ) being very old, while a younger component (2-4 Gyr) contributes $20 \%-30 \%$ (Rejkuba et al. 2011). The GCS is well investigated regarding kinematics, ages, and abundances (Peng et al. 2004a,b; Woodley et al. 2010b,a). Most GCs are old, but there are also intermediate-age and 
younger clusters. The kinematics of both planetary nebulae and GCs indicate the existence of a massive dark halo (Woodley et al. 2010b). Cen A is a "double-double" radio galaxy (e.g. Saikia \& Jamrozy 2009) with probably recurrent nuclear activity.

Next in distance to Cen A is NGC 1316 (Fornax A) in the outskirts of the Fornax cluster. There is a vast quantity of literature on NGC 1316 with studies in many wavelength bands. We give a representative overview.

The morphology of NGC 1316 is very different from Cen A and is characterized by an inner elliptical body with a lot of fine dust structure in its central region, best admired in Hubble Space Telescope (HST) images, and an extended elongated structure with loops, tails, and tidal arms that cover an area on the sky almost comparable to the full moon. These were first described by Schweizer (1980) in a wide-field photographic study. Schweizer (1981) also noted the high central surface brightness. Furthermore, he performed kinematical measurements and found an abnormally low $M / L_{B}=1.8$, which in part is a consequence of his large adopted distance of $32.7 \mathrm{Mpc}$. More modern values are higher, e.g. $M / L_{V}=2.5$ (Shaya et al. 1996) and $M / L_{K_{\mathrm{s}}}=0.65$ (Nowak et al. 2008), but still clearly indicate an intermediate-age population. Schweizer (1980), moreover, detected an inner ionizing rotating disk and a giant HII-region south of the center. He suggested a merger that is supposed to have occurred between 0.5 and 2 Gyr ago.

Mackie \& Fabbiano (1998), on the base of deep $B$-band imaging and imaging in $\mathrm{H}_{\alpha}$ and NII, revisited the morphology and studied the distribution of gaseous line emission. They detected faint emission to the north, between the nucleus and the companion galaxy NGC 1317. They also detected the interesting "extended emission line region (EELR)", an elongated feature of length $1.5^{\prime}$ southwest to the nucleus at a distance of about $6^{\prime}$, in a region without any signature of ongoing star formation. Using ROSAT data, they detected hot gas apparently associated with Schweizer's tidal features L1 and L2 (see Fig. 2 of Schweizer 1980). Their general assessment of NGC 1316's history is a diskdisk or disk-E merger older than $1 \mathrm{Gyr}$ and a smaller merger about 0.5 Gyr ago.

Horellou et al. (2001) studied the content of atomic and molecular gas. Molecules are abundant in the central region, but HI has been detected only in some single spots, among them the EELR and the southern HII-region.

NGC 1316 has been observed in the X-rays with practically all X-ray satellites (Einstein: Fabbiano et al. 1992; ROSAT: Feigelson et al. 1995, Kim et al. 1998; ASCA: Iyomoto et al. 1998). More recently, Kim \& Fabbiano (2003) used Chandra to constrain the temperature of the hot interstellar medium (ISM) $(\approx 0.6 \mathrm{keV})$, to detect a low-luminosity AGN, and to detect 96 $\mathrm{X}$-ray point sources. These authors quote a range $0.25 Z_{\odot}<$ $Z<1.3 Z_{\odot}$ for the metal-abundance of the ISM. The values derived from Suzaku-data are lower than solar (Konami et al. 2010, e.g. Fe is only 0.44 solar). NGC 1316 is also among the sample of elliptical galaxies of Fukazawa et al. (2006), who quote X-ray based dynamical masses. Nagino \& Matsushita (2009) used XMM-Newton to constrain the gravitational potential.

Kinematics of the stellar population and abundances have been studied by Kuntschner (2000), Thomas et al. (2005), and Bedregal et al. (2006). The luminosity-weighted abundance is higher than solar $(Z \approx 0.03)$.

Arnaboldi et al. (1998) presented kinematics of 43 planetary nebulae and long-slit spectroscopy along the major and the minor axis. They analyzed the velocity field and gave some dynamical considerations, resulting in a total mass

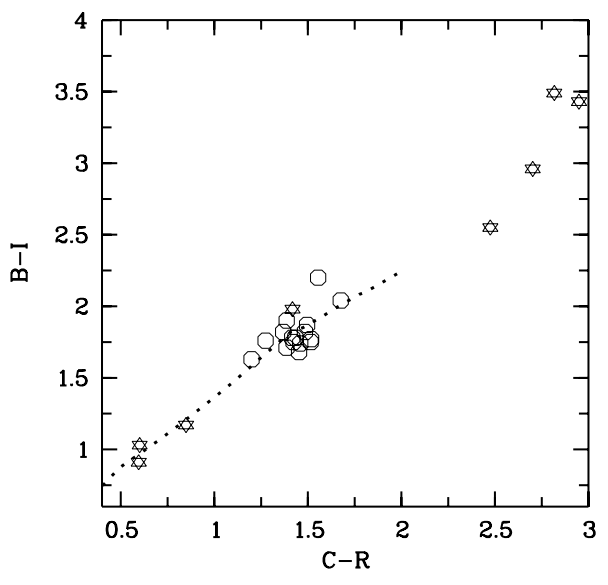

Fig. 1. Comparison of our $C-R$ colors with the $B-I$ colors of Goudfrooij et al. (2001a) for stars and confirmed globular clusters in NGC 1316. Star symbols denote stars, circles denote clusters. The dotted line delineates theoretical integrated single stellar population colors for solar metallicity, taken from Marigo et al. (2008).

of $2.9 \times 10^{11} M_{\odot}$ and a mass-to-light ratio in the $B$-band of 8 within $16 \mathrm{kpc}$.

Nowak et al. (2008) investigated the innermost kinematics to constrain the mass of the supermassive black hole. They found a mass of about $1.5 \times 10^{8} M_{\odot}$, marginally consistent with the masssigma relation from Tremaine et al. (2002). Noteworthy is the double giant radio lobe with centers in projected galactocentric distances of about $100 \mathrm{kpc}$ (e.g. see Fig. 1 of Horellou et al. 2001). Lanz et al. (2010) used mid-infrared and X-ray data to develop a scenario in which the lobes have been created by a nuclear outburst about 0.5 Gyr ago.

Very recently, McNeil-Moylan et al. (2012) presented radial velocities of almost 800 planetary nebulae in NGC 1316, one of the largest samples so far. A spherical Jeans model indicates a high dark matter content, characterized by a dark halo with a large core.

The GCS has been investigated several times with various intentions. Apparently, the first observations devoted to GCs were made with HST (WF/PC-I, Shaya et al. 1996). These authors list 20 clusters in the very central region. One object has $M_{V}=-12.7$ which, if old, today would be called an ultracompact dwarf (e.g. Mieske et al. 2008).

More work on GCs, using the HST and the NTT, was presented by Goudfrooij et al. (2001a,b, 2004). Based on infrared colors, they found ages for the brightest clusters consistent with 2-3 Gyr. The luminosity function (LF) of red GCs was found to be a power law with the exponent -1.2 , flatter than that of normal ellipticals, while the blue clusters exhibit the normal LF with a turn-over at about $M_{V} \approx-7.2$ (but see our remarks in Sect. 8.3).

At the same time, Gómez et al. (2001) studied the GCS photometrically, using images obtained with the $3.6 \mathrm{~m}$ telescope on La Silla, ESO, in $B, V, I$. They did not detect a clear bimodality but a difference in the azimuthal distribution of blue and red clusters in the sense that the red clusters follow the ellipticity of the galaxy's bulge, while the blue clusters are more circularly distributed. Moreover, they confirmed the very low specific frequency previously found by Grillmair et al. (1999) of $S_{N}=0.4$.

Our intention is to study the GCS on a larger field than has been done before. Moreover, our Washington photometry permits a useful comparison to the Washington photometry of elliptical galaxies, obtained with the same instrumentation 
(Dirsch et al. 2003a,b, 2005; Bassino et al. 2006a). We adopt a distance of $17.8 \mathrm{Mpc}$, quoted by Stritzinger et al. (2010) using the four type Ia supernovae that have so far appeared in NGC 1316. The high supernova rate is an additional indicator for an intermediate-age population. The surface brightness fluctuation distance (Blakeslee et al. 2009) is $21 \pm 0.6 \mathrm{Mpc}$ which may indicate a still unsolved zero-point problem.

This paper is the first in a series devoted to NGC 1316 and its globular cluster system. Future papers will treat the kinematics and dynamics of the GCS as well as SH2, the HII-region detected by Schweizer (1980).

\section{Observations and reductions}

\subsection{Data}

Since the present data have been taken and reduced together with the data leading to the Washington photometry of NGC 1399 (Dirsch et al. 2003b), all relevant information regarding the reduction technique can be found in the previous publication. We therefore give the most basic information only.

The data set consists of Washington wide-field images of NGC 1316 taken with the MOSAIC camera mounted at the prime focus of the CTIO $4 \mathrm{~m}$ Blanco telescope during the night 20/21 November 2001 (the entire run had three nights). We used the Kron-Cousins $R$ and Washington $C$ filters. Although the genuine Washington system uses T1 instead of $R$, Geisler (1996) has shown that the Kron-Cousins $R$ filter is more efficient than T1, because of its wider bandwidth and higher throughput. He also showed that $R$ and T1 magnitudes are closely related, with only a very small color term and a zero-point difference of 0.02 mag.

The MOSAIC wide-field camera images a field of $36^{\prime} \times 36^{\prime}$, with a pixel scale of $0.27^{\prime \prime} /$ pixel. The observations were performed in the 16 channel read-out mode. We obtained three R-exposures with an exposure time of $600 \mathrm{~s}$ each, and three C-exposures with an exposure time of 1200 s each. Additionally, we observed standard stars for the photometric calibration. In the following, we refer to colors as $C-R$ and not as C-T1, although the calibration provides Washington colors. The $\mathrm{C}$-images have not been dithered.

The seeing was about $1^{\prime \prime}$ in the $R$-images and $1.5^{\prime \prime}$ in the C-images.

\subsection{MOSAIC reduction and photometry}

The MOSAIC data were reduced using the mscred package within IRAF. In particular this software is able to correct for the variable pixel scale across the CCD, which would otherwise cause a 4 percent variability of the brightness of stellar-like objects from the center to the corners. The flatfielding resulted in images that had remaining sensitivity variations of about $1.5 \%$. In particular Chip 4 and Chip 5 showed discernible remaining flatfield structure (but within the given deviation).

The actual photometry was performed with DAOPHOT II.

\subsection{Photometric calibration}

Standard fields for the photometric calibration were observed in each of the three nights. The weather conditions were photometric. We observed 4-5 fields, each containing about ten standard stars from the list of Geisler (1996), with a large coverage of airmasses (typically from 1.0 to 1.9 ). It was possible to use a single transformation for all three nights, since the coefficients derived for the different nights were indistinguishable within the errors. We derived the following relations between instrumental and standard magnitudes:

$$
\begin{aligned}
R=R_{\text {inst }}+ & (0.72 \pm 0.01)-(0.08 \pm 0.01) X_{R} \\
& +(0.021 \pm 0.004)(C-R) \\
C=C_{\text {inst }}+ & (0.06 \pm 0.02)-(0.30 \pm 0.01) X_{C} \\
+ & (0.074 \pm 0.004)(C-R) .
\end{aligned}
$$

The standard deviation of the difference between our calibrated and tabulated magnitudes is $0.018 \mathrm{mag}$ in $R$ and $0.027 \mathrm{mag}$ in $C$.

To calibrate the NGC 1316 field we identified isolated stars that were used to determine the zero points. The scatter between the zero points determined from individual stars is $0.03 \mathrm{mag}$ and most probably due to flat-field uncertainties.

The final uncertainties of the zero points are $0.03 \mathrm{mag}$ and $0.04 \mathrm{mag}$ for $R$ and $C$, respectively. This results in an absolute calibration uncertainty in C-T1 of $0.05 \mathrm{mag}$ (the uncertainty in the color term can be neglected). See, however, Fig. 1 which indicates that the calibration is quite precise in the interval $0.5<C-R<2.5$.

The foreground reddening toward NGC 1316 according to Schlegel et al. (1998) is $E_{B-V}=0.02$. Using $E_{C-T 1}=1.97 E_{B-V}$ (Harris \& Canterna 1977) we had to correct $C-R$ by 0.04 mag. In the following we neglect the foreground reddening, since no conclusion depends strongly on an absolute precision of this order.

\subsection{Comparison with previous photometry}

We now compare our photometry for confirmed clusters in NGC 1316 with the $B-I$ photometry of Goudfrooij et al. (2001a). There are 17 clusters and 10 stars for which $B-I$ and $C-R$ colors exist. Our point-source selection excludes the object 211 of Goudfrooij et al., which seems to be very extended. Figure 1 shows the comparison. Star symbols denote stars, circles denote clusters. For $C-R$ colors bluer than 2, the agreement is very satisfactory with the exception of one cluster (the object 115 in Goudfrooij et al.'s list). This cluster is projected onto the immediate vicinity of a dust patch, which may have influenced its photometry. The stars redder than $C-R=2.5$ deviate significantly, which plausibly shows a deficiency of the photometric calibration, which may be not valid for such red objects. The dotted line represents the theoretical integrated single stellar population colors for solar metallicity, taken from Marigo et al. (2008). Again, the agreement is very satisfactory. All clusters (except 115 of Goudfrooij et al.) are located outside of the inner dust structures. However, only a strong reddening would be detectable. The reddening vector in Fig. 1 is $E(B-I)=$ $1.18 E(C-R)$, adopting the reddening law of Harris \& Canterna (1977), i.e. almost parallel to the $(B-I)-(C-R)$-relation.

\subsection{Photometric uncertainties and selection of point sources}

Figure 2 shows the photometric errors as given by DAOPHOT for all sources. The upper panel shows the uncertainties in $R$, the lower panel in $C-R$. The point sources and resolved sources are clearly separated for magnitudes fainter than $R=18$. Brighter point sources are saturated. The uncertainties in color grow quickly for $R>24 \mathrm{mag}$. However, we aimed to select GC candidates not only on account of photometric uncertainties, but by goodness of fit and degree of resolution through the ALLSTAR parameters chi and sharp.

These parameters are plotted in Fig. 3 with sharp in the upper panel and chi in the lower panel. Positive values of sharp indicate 


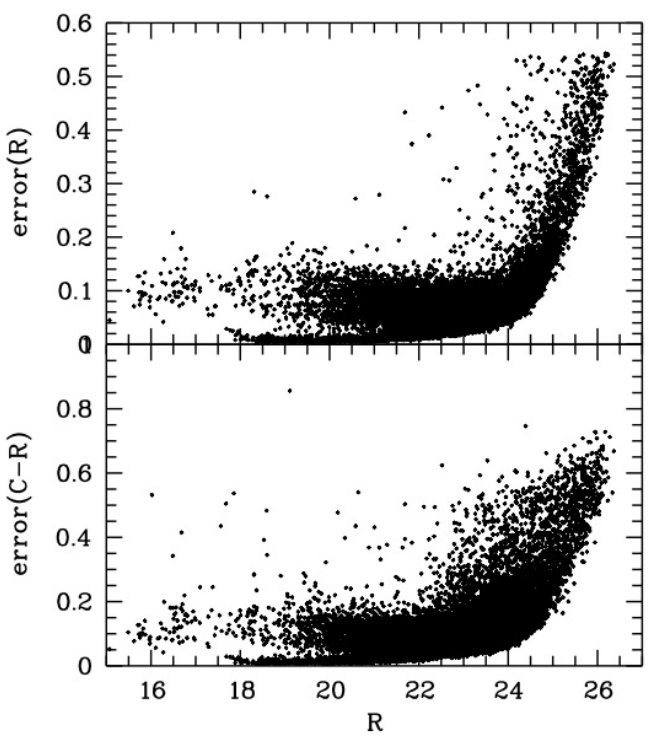

Fig. 2. Photometric uncertainties for the entire photometric catalog as given by DAOPHOT in $R$ (upper panel) and $C-R$ (lower panel). This plot shows the full range of uncertainties. Most objects are extended and appear with relatively large uncertainties also at bright magnitudes. The retained point sources are those with very small errors.

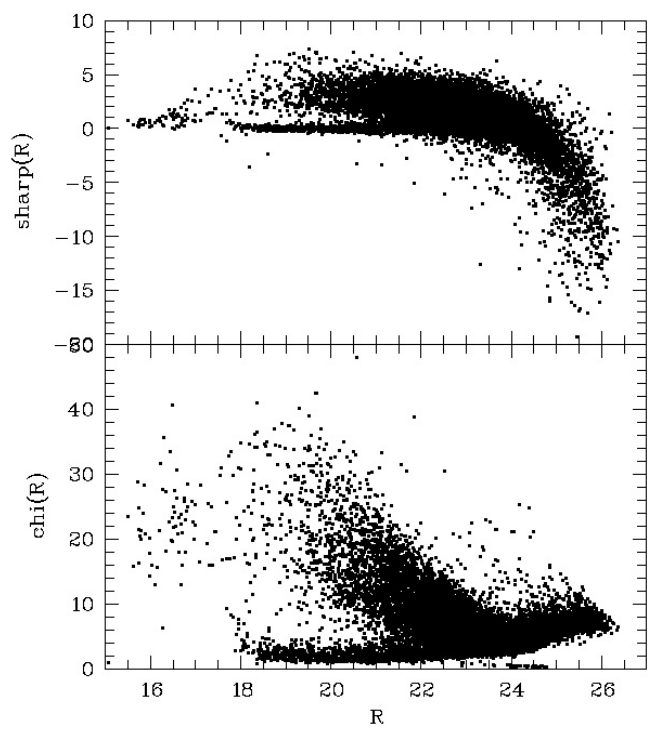

Fig. 3. Chi- and sharp parameters for the entire photometric catalog as given by DAOPHOT. We selected as point sources objects within the sharp-parameter interval $-0.5<$ sharp $<0.8$ and chi $<4$.

resolved objects, negative values deficiencies in the photometry, mainly for the faintest objects. The unsaturated resolved objects are of course galaxies, but we cannot exclude that some extended GCs are among them (see the remark in Sect. 2.4).

Guided by Fig. 3, we selected as point sources objects within the sharp-parameter interval $-0.5<$ sharp $<0.8$ and chi less than 4 . We used the $R$-image because of its better seeing. Furthermore, we selected the magnitude interval $18<R<24$ and the uncertainties in $R$ and $C-R$ to be less than $0.1 \mathrm{mag}$ and $0.2 \mathrm{mag}$, respectively. This selection reduced the full sample of about 22000 objects found in the entire field to 4675 . The resulting photometric errors for the point sources are shown in Fig. 4.

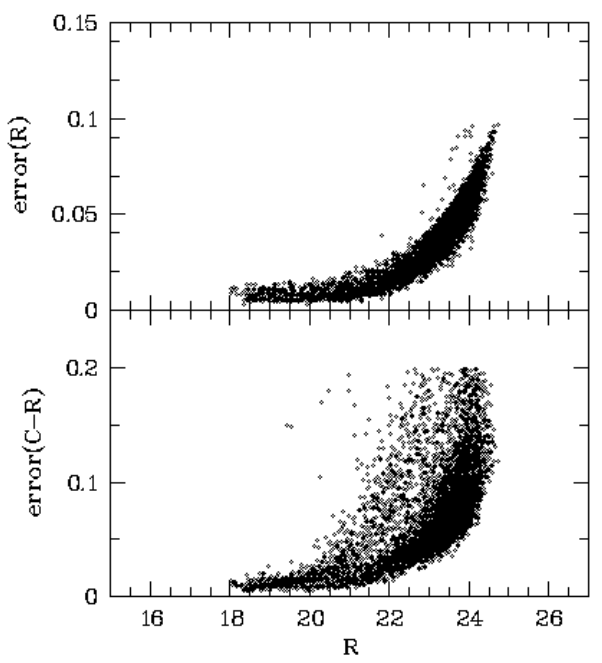

Fig. 4. Photometric uncertainties of selected point sources. Since the $\mathrm{C}$-images are not dithered, the larger errors at a given mag are explainable by sources lying close to the CCD-gaps.

\section{The CMD of point sources}

The CMD of the selected 4675 point sources is shown in Fig. 5 (left panel) together with their location (in pixels; right panel). Visible are the vertical stellar sequences at $C-R=0.7$ and $C-R=3$. The bulk of GC candidates are found between $C-R=1$ and $C-R=1.8$ for $R$-magnitudes fainter than 20. There may also be GCs brighter than $R=20$. The bulge of NGC 1316 is striking. We recall that the optically visible full diameter of NGC 1316 is about $27^{\prime}$, corresponding to 6000 pixels. At first glance there is no bimodality. A more detailed look into color intervals will modify this impression. $R=18$ mag is approximately the saturation limit for point sources. A comparison with the field around NGC 1399 (Dirsch et al. 2003b, their Fig. 3) shows that the present data are considerably deeper. At a magnitude level of $R=24 \mathrm{mag}$, the incompleteness due to faint sources in $\mathrm{C}$ starts at $C-R=1.3$, while around NGC 1316, it starts at $C-R=2.3$.

The inner blank region of NGC 1316 shows the incompleteness where the galaxy light becomes too bright. To avoid this incompleteness region, we shall consider only GC candidates with distances larger than $2^{\prime}$.

Figure 6 shows CMDs with three different radius selections (see figure caption). In the inner region (which in fact is blank within the inner 30"; here we consider also clusters closer to the center than $2^{\prime}$ ) the vertical sequence of bright objects at $C-R=1.5$ is striking. Some of these GC candidates are already confirmed clusters from the radial velocity sample of Goudfrooij et al. (2001b). Very interesting is the blue object with $C-R=0.64$ (object 119 in Goudfrooij et al.'s list), which must be quite young, about 0.5 Gyr (see Sect. 8.3), assuming solar metallicity. Two more objects with very red colors, which appear as GCs in the list of Goudfrooij et al. (numbers 122 and 211), are not marked. With $R=20.37, C-R=3.26$, and $R=20.77, C-R=3.57$, they fall drastically outside the color range of GCs. They are not obviously associated with dust. On our FORS2/VLT pre-images (seeing about $0.5^{\prime \prime}$ ), used for the spectroscopic campaign, they are clearly resolved and might be background galaxies, in which case the velocities are incorrect.

However, if one bright young cluster exists, we also expect fainter ones of similar age. We show this statistically. The middle panel demonstrates that the occurrence of these bright clusters 

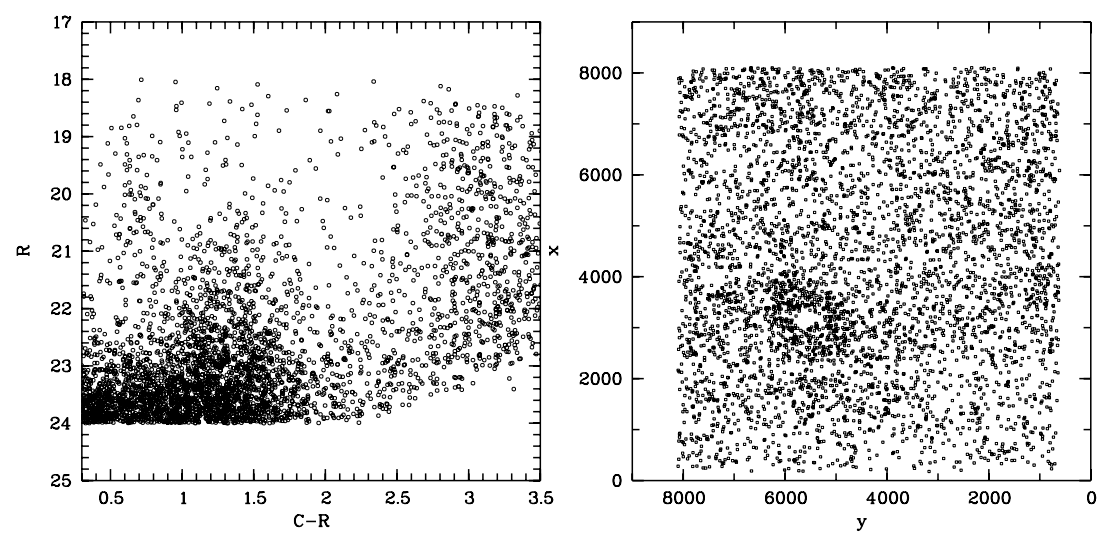

Fig. 5. Left panel: the CMD for 4675 point sources in the MOSAIC field around NGC 1316. The bulk of the GCs are found in the color interval $1<C-R<2$, but there are also bluer GCs. However, most point sources bluer than $C-R=1$ and $23<R<24$ are unresolved background galaxies. Right panel: distribution of point sources in our MOSAIC field. The inner galaxy bulge is very clearly visible. The size is $34^{\prime} \times 34^{\prime}$. North is up, east to the left.

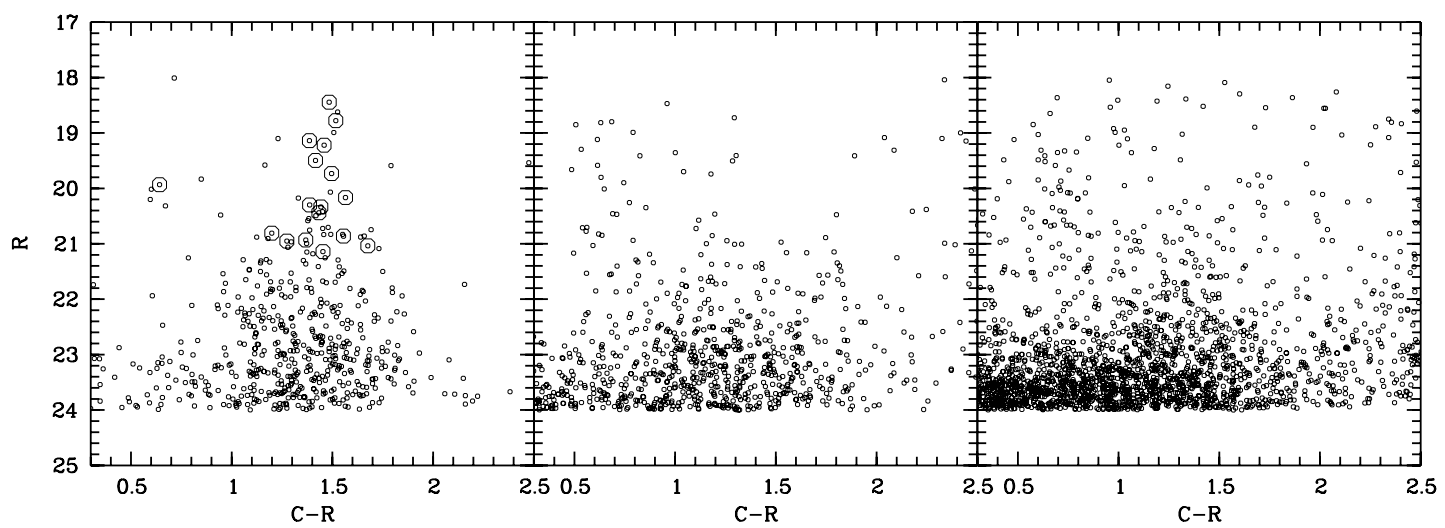

Fig. 6. The three CMDs refer to different radius selections. The left panel shows GC candidates within the inner $4^{\prime}$, the middle panel between $4^{\prime}$ and $10^{\prime}$, and the outer panel at radii larger than $10^{\prime}$. The respective area sizes then are related as 1:5.2:25.3. The inner CMD is thus dominated by genuine GCs. In the left panel, spectroscopically confirmed GCs (Goudfrooij et al. 2001b) are marked. Note the blue object at $C-R=0.64$, which must be a quite young cluster.

is restricted to the inner region, but there are still overdensities of GC candidates. The right panel shows almost exclusively foreground stars and background galaxies. We now look closer at the distribution of colors.

\section{Color distribution}

Figure 7 displays the color distribution in different radial regimes (left: radius $<10^{\prime}$; middle: $4^{\prime}<$ radius $<10^{\prime}$; right: $2<$ radius $<$ $4^{\prime}$ ) for all magnitudes ( $R<24$ mag, lower row), for bright magnitudes $(R<22$, upper row $)$, and for an intermediate sample magnitudes $\left(R<23\right.$, middle row). A radius of $4^{\prime}$ approximately marks the extension of the bulge. The ordinates are given in numbers per $\operatorname{arcmin}^{2}$. The corresponding values and their uncertainties are given in Table 1 for $R<24$ mag and in Table 2 for $R<23$ mag.

The counts are background-corrected, the background being evaluated outside $13^{\prime}$.

The main observations from Fig. 7 are:

- the entire system shows a continuous distribution of colors with no sign of bimodality;

- the peak of the color distribution is displaced blueward with respect to the galaxy color, more so for the outer clusters;

- the inner bright clusters show a pronounced bimodality with peaks at $C-R=1.4$ and $C-R=1.1$. These peaks are
Table 1. Background-subtracted numbers per $\operatorname{arcmin}^{2}$ of GC candidates brighter than $R=24$ mag in color bins and for different radial selections.

\begin{tabular}{ccccc}
\hline \hline Color & Background & $2<r<10$ & $4<r<10$ & $2<r<4$ \\
\hline 0.650 & $0.144 \pm 0.014$ & $0.041 \pm 0.028$ & $0.026 \pm 0.029$ & $0.148 \pm 0.089$ \\
0.750 & $0.167 \pm 0.015$ & $-0.01 \pm 0.026$ & $-0.01 \pm 0.028$ & $-0.03 \pm 0.061$ \\
0.850 & $0.155 \pm 0.014$ & $0.033 \pm 0.028$ & $0.023 \pm 0.030$ & $0.111 \pm 0.085$ \\
0.950 & $0.167 \pm 0.015$ & $0.085 \pm 0.032$ & $0.064 \pm 0.033$ & $0.231 \pm 0.104$ \\
1.050 & $0.159 \pm 0.015$ & $0.141 \pm 0.034$ & $0.083 \pm 0.034$ & $0.398 \pm 0.122$ \\
1.150 & $0.163 \pm 0.015$ & $0.216 \pm 0.038$ & $0.113 \pm 0.035$ & $0.766 \pm 0.158$ \\
1.250 & $0.156 \pm 0.014$ & $0.214 \pm 0.037$ & $0.089 \pm 0.034$ & $0.693 \pm 0.151$ \\
1.350 & $0.138 \pm 0.014$ & $0.225 \pm 0.037$ & $0.084 \pm 0.032$ & $0.896 \pm 0.166$ \\
1.450 & $0.121 \pm 0.013$ & $0.226 \pm 0.036$ & $0.087 \pm 0.031$ & $0.860 \pm 0.162$ \\
1.550 & $0.098 \pm 0.011$ & $0.176 \pm 0.032$ & $0.053 \pm 0.026$ & $0.777 \pm 0.153$ \\
1.650 & $0.077 \pm 0.010$ & $0.137 \pm 0.028$ & $0.063 \pm 0.025$ & $0.613 \pm 0.136$ \\
1.750 & $0.058 \pm 0.009$ & $0.076 \pm 0.022$ & $0.025 \pm 0.020$ & $0.393 \pm 0.110$ \\
1.850 & $0.047 \pm 0.008$ & $0.077 \pm 0.021$ & $0.055 \pm 0.021$ & $0.165 \pm 0.075$ \\
1.950 & $0.036 \pm 0.007$ & $0.015 \pm 0.015$ & $0.005 \pm 0.014$ & $0.070 \pm 0.054$ \\
2.050 & $0.054 \pm 0.009$ & $-0.01 \pm 0.014$ & $-0.00 \pm 0.016$ & $-0.02 \pm 0.028$ \\
2.150 & $0.036 \pm 0.007$ & $0.021 \pm 0.015$ & $0.009 \pm 0.015$ & $0.096 \pm 0.060$ \\
\hline
\end{tabular}

Notes. The bin widths are $0.1 \mathrm{mag}$. The background is defined by $r>13^{\prime}$. The uncertainties are based on the square root of the raw counts. Negative values are kept for formal correctness.

already indicated in the small, but clean, spectroscopic sample of Goudfrooij et al. (2001a);

- the outer clusters show only a peak a $C-R=1.1$; 


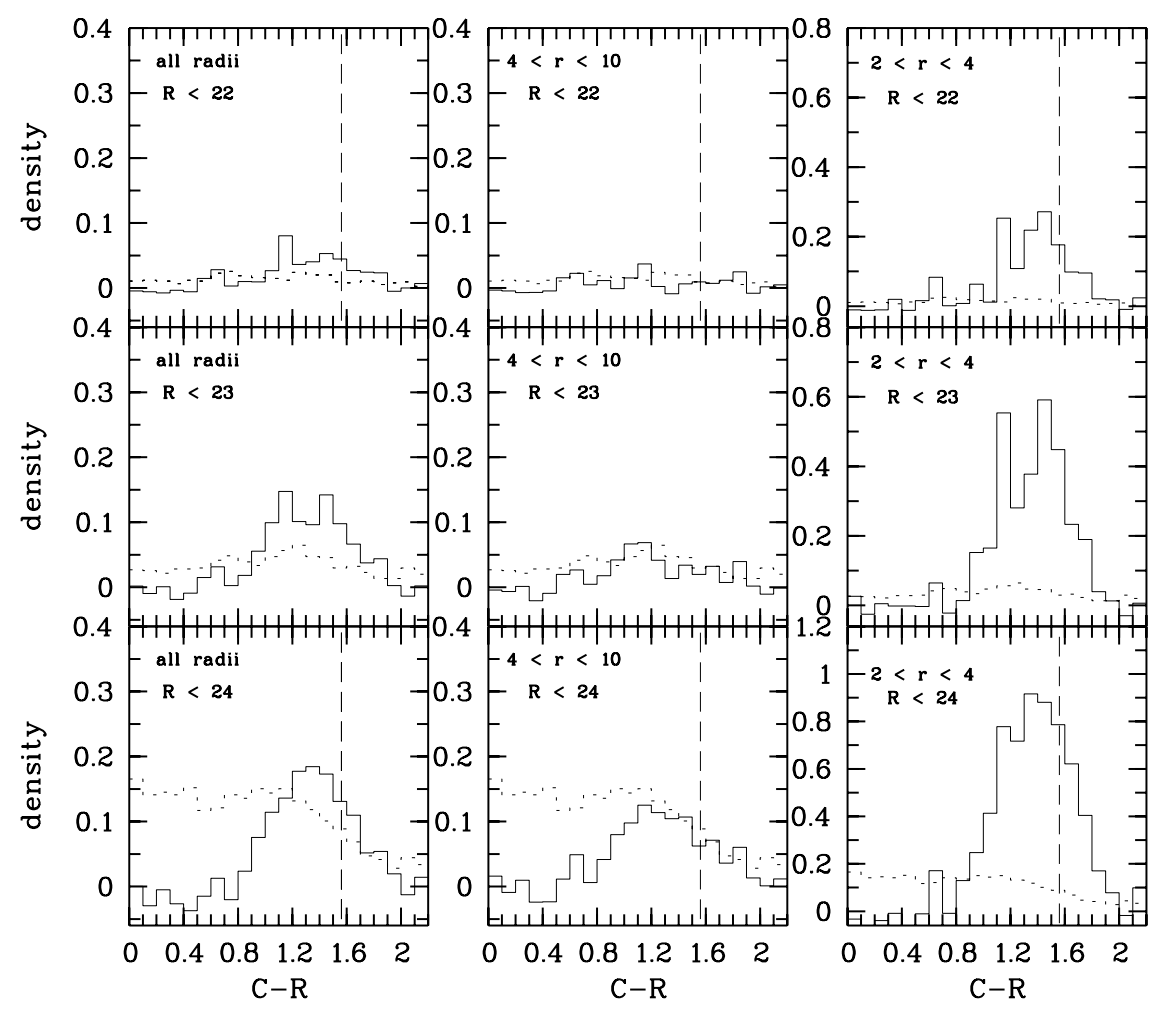

Fig. 7. Color histograms, corrected for background counts, for three radial regimes and for different magnitude ranges. Left panel: 0-10 arcmin, middle panel: 4-10 arcmin, right panel: 2-4 arcmin. The upper row displays magnitudes brighter than $R=22$, the middle row objects brighter than $R=23$, the lower row objects brighter than $R=24$. The respective backgrounds are indicated by the dotted histograms. The vertical dashed line indicates the color of NGC 1316 at $2^{\prime}$, outside the dusty region.

Table 2. Background-subtracted numbers per $\operatorname{arcmin}^{2}$ of GC candidates brighter than $R=23$ mag in color bins and for different radial selections.

\begin{tabular}{ccccc}
\hline \hline Color & Background & $2<r<10$ & $4<r<10$ & $2<r<4$ \\
\hline 0.650 & $0.042 \pm 0.007$ & $0.032 \pm 0.017$ & $0.026 \pm 0.018$ & $0.064 \pm 0.054$ \\
0.750 & $0.048 \pm 0.008$ & $0.003 \pm 0.015$ & $0.004 \pm 0.016$ & $-0.02 \pm 0.028$ \\
0.850 & $0.039 \pm 0.007$ & $0.018 \pm 0.015$ & $0.018 \pm 0.016$ & $0.014 \pm 0.038$ \\
0.950 & $0.034 \pm 0.007$ & $0.056 \pm 0.018$ & $0.042 \pm 0.018$ & $0.152 \pm 0.071$ \\
1.050 & $0.047 \pm 0.008$ & $0.099 \pm 0.023$ & $0.066 \pm 0.022$ & $0.165 \pm 0.075$ \\
1.150 & $0.056 \pm 0.009$ & $0.147 \pm 0.027$ & $0.068 \pm 0.023$ & $0.554 \pm 0.128$ \\
1.250 & $0.065 \pm 0.009$ & $0.101 \pm 0.025$ & $0.041 \pm 0.022$ & $0.280 \pm 0.096$ \\
1.350 & $0.047 \pm 0.008$ & $0.096 \pm 0.023$ & $0.013 \pm 0.017$ & $0.377 \pm 0.106$ \\
1.450 & $0.046 \pm 0.008$ & $0.142 \pm 0.026$ & $0.034 \pm 0.019$ & $0.591 \pm 0.130$ \\
1.550 & $0.030 \pm 0.006$ & $0.098 \pm 0.021$ & $0.020 \pm 0.015$ & $0.448 \pm 0.113$ \\
1.650 & $0.032 \pm 0.007$ & $0.066 \pm 0.019$ & $0.032 \pm 0.017$ & $0.233 \pm 0.084$ \\
1.750 & $0.023 \pm 0.006$ & $0.038 \pm 0.015$ & $0.007 \pm 0.012$ & $0.189 \pm 0.075$ \\
1.850 & $0.013 \pm 0.004$ & $0.044 \pm 0.014$ & $0.039 \pm 0.015$ & $0.040 \pm 0.038$ \\
1.950 & $0.013 \pm 0.004$ & $0.002 \pm 0.008$ & $0.002 \pm 0.009$ & $0.013 \pm 0.027$ \\
2.050 & $0.030 \pm 0.006$ & $-0.01 \pm 0.010$ & $-0.01 \pm 0.011$ & $-0.03 \pm 0.006$ \\
2.150 & $0.020 \pm 0.005$ & $0.002 \pm 0.010$ & $-0.00 \pm 0.010$ & $0.006 \pm 0.027$ \\
\hline
\end{tabular}

Notes. The bin widths are $0.1 \mathrm{mag}$. The background is defined by $r>13^{\prime}$. The uncertainties are based on the square root of the raw counts. Negative values are kept for formal correctness.

- there is evidence for a small GC population bluer than $C-R=1.0$, which is approximately the metal-poor limit for old clusters (see Fig. 14).

The color distribution of GCs in a typical elliptical galaxy in our photometric system is bimodal with two peaks at $C-R=1.35$ and $C-R=1.75$ (Bassino et al. 2006b) with little scatter. A
Gaussian fit to the entire sample in the color range 0.8-2 gives a peak color of $1.33 \pm 0.01$ and a sigma of $0.3 \pm 0.01$, which resembles indeed the position of the "universal" blue peak color in the GCS of elliptical galaxies, while the distribution is broader. On first sight, this is somewhat surprising since we expect quite a different composition of GCs: there should be a mixture of old GCs from the pre-merger components, and an unknown fraction of GCs, presumably a large one, which stem from one or more star-bursts. Later star-forming events with GC production might also have occurred. That the already confirmed clusters define such a sharp color peak (Fig. 6) and that the existence of intermediate-age clusters has already been shown strongly suggests that intermediate-age objects are dominating among the bright clusters. Older clusters are then cumulatively mixed in with decreasing brightness.

The peak color for the outer sample is $1.23 \pm 0.037$ and $1.37 \pm 0.01$ for the inner sample. This difference must, in part, be caused by a different composition of GC populations because the density of red clusters shows a steeper decline, but we cannot exclude a contribution from differential reddening. However, this must be small since there are hardly any cluster candidates redder than $C-R=1.9$. Moreover, the excellent reproduction of the theoretical color-color relations in Fig. 1 indicates the absence of strong reddening for this inner sample where we would expect to see reddening effects first.

\section{Spatial distribution of clusters}

\subsection{Density profile and total numbers}

Given the complicated morphology and a probable mixture of bulge and disk symmetry, a surface density profile for GCs 


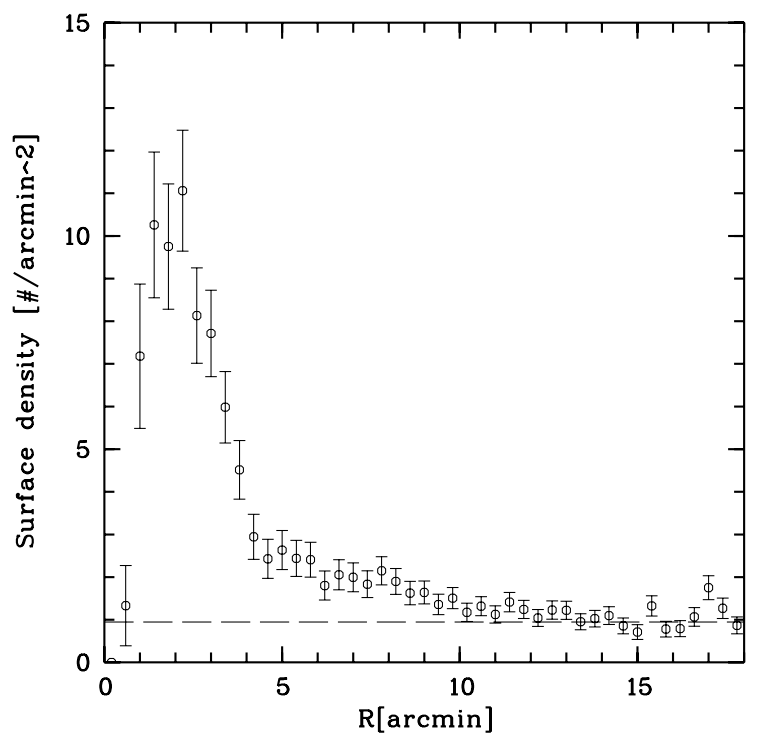

Fig. 8. Surface densities for selected point sources. The dashed horizontal line indicates the background. Inside $2^{\prime}$, the counts are severely affected by incompleteness. The profile seems to be inflected at a radius of $4^{\prime}$. We define the background by counts outside $13^{\prime}$.

in NGC 1316 will not have the same meaning as in spherical elliptical galaxies, where deprojection is possible. We instead used the density profile to evaluate the total extent of the cluster system and possible features in the profile. For convenience we counted in circular annuli and selected point sources in the color range $0.9-2$ according to the above selection. The full annuli become sectors for distances larger than $10^{\prime}$, when the image border is reached. We corrected for this geometrical incompleteness.

First we show the general density profile, then the twodimensional distribution in various color intervals, and finally the radial density profiles in these intervals.

Figure 8 shows the resulting surface densities. Inside $2^{\prime}$ the counts are severely affected by incompleteness due to the galaxy light and possible extinction by dust. Outside $13^{\prime}$ the counts are not distinguishable anymore from the background, for which we determine 0.946 objects/arcmin ${ }^{2}$.

Summing up the bins in Fig. 8, we find $636 \pm 35$ as the total number of GC candidates down to our magnitude limit of $R=24$ and in the color range $0.9<C-R<2.0$ inside $13^{\prime}$. Correcting for the inner incompleteness would not enhance this number considerably. For an old GCS of a normal elliptical galaxy with a Gaussian-like luminosity function and a turn-over magnitude corresponding to its distance, one would roughly double this number to have a fair estimation of the total number. Since a single turn-over magnitude, valid for the entire cluster system, does not exist, one would underestimate this number. About 1400-1500 clusters seems to be a good guess, somewhat larger than the number given by Gómez et al. (2001). In any case, it is a relatively poor cluster system. We return to this point in the discussion.

This radius of $13^{\prime}$ agrees well with the extension of NGC 1316 seen in Fig. A.1, which we expect if the extension of NGC 1316 is determined by the dynamical processes during the merger event(s). One notes an inflection point in Fig. 8 at about $4^{\prime}$. Its nature becomes clearer if we subtract the background and plot surface densities for different color intervals. This is done in Fig. 10 in a linear display, including the interval $0.8<C-R<1.1$, and in Fig. 11 in double-logarithmic display.
The blue population is statistically well visible, although poor. The GC candidates in the color interval 1.3-1.6 show a sharp decline at $4^{\prime}$, which defines the bulge. The outer clusters in this sample are mainly located to the south.

\subsection{The two-dimensional distribution of clusters}

NGC 1316 exhibits an elliptical symmetry only in its inner parts. At larger radii, the overall morphology is characterized by tidal(?) tails and loops. It is now interesting to ask whether morphological features of NGC 1316 can be found back in the distribution of GCs. Naturally, one does that in color intervals to suppress the background and (hopefully) obtain insight into the nature of the cluster candidates.

We plausibly assume that intermediate-age and younger clusters have at least the metallicity of the stellar bulge, i.e. at least solar metallicity. We refer to Fig. 14 for a theoretical relation between age and Washington colors.

Figure 9 shows the two-dimensional distribution of objects in six different color-intervals.

Interval $0.4<C-R<0.8$.

Here we expect younger clusters with ages about 0.5 Gyr or somewhat younger. NGC 1316 is statistically not visible but note the cluster with $C-R=0.6$ in Fig. 6. We anticipate (Richtler et al., in prep.) that we identified another object with $C-R=0.4$ by its radial velocity. Certainly there are not many bright clusters within this age range and they do not seem to belong to a particularly strong star formation epoch.

Interval $0.8<C-R<1.1$.

Now the field of NGC 1316 becomes recognizable. With solar metallicity or higher, ages are between 0.6 and 1 Gyr. Noteworthy are the clumps roughly $1.5^{\prime}$ to the NE and a slight overdensity, which is projected roughly onto the L1-feature.

Interval $1.1<C-R<1.3$.

In this interval we expect old, metal-poor clusters and younger clusters between 1 and 1.5 Gyr. NGC 1316 is now clearly visible. The major axis of the distribution seems to show a position angle of about $90^{\circ}$, different from the main body. However, the more quantitative source counts in dependence on azimuth still show an overdensity at the position angle of the major axis of NGC 1316 toward the northeast, while the peak towards the southwest is less pronounced (Fig. 12). The fraction of intermediate-age clusters is unknown. We discuss this issue in more detail in Sect. 8.5, where we argue that younger clusters indeed provide the dominating population.

Interval $1.3<C-R<1.6$.

This interval samples the maximum of the color distribution. One notes the sharp definition of the galaxy's luminous body. Perpendicular to the major axis the cluster reaches the background at about $2.5^{\prime}$. A gap is visible at a distance of approximately $4^{\prime}$ to the south. Even farther to the south, the cluster candidates populate a region roughly defined by Schweizer's L1-structure. Note also the horizontal boundary of cluster candidates at a distance of $8^{\prime}$ which nicely delineates the sharp ridge in NGC 1316.

Interval $1.6<C-R<1.9$.

Here we expect the majority of the metal-rich GCs of the premerger components. That NGC 1316 is still well visible indicates a similar concentration as that of the metal-poor clusters. The overall symmetry follows the main body of NGC 1316, which indicates the presence of an old population. Indeed, as we argue in Sect. 8.6, this population dominates the bulge mass. 

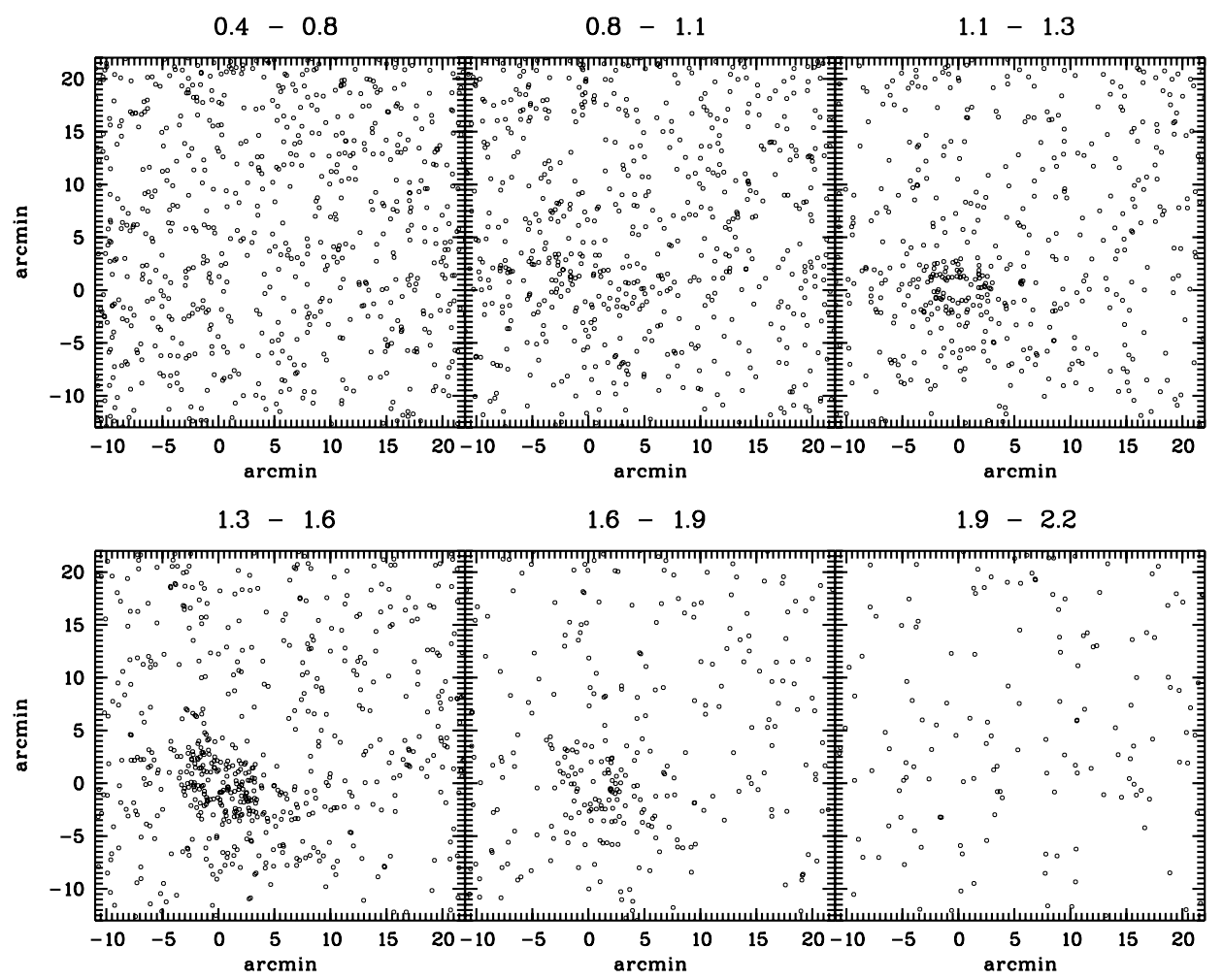

Fig. 9. Two-dimensional distribution of GC candidates in six $C-R$ color intervals. North is up, east to the left. The origin is NGC 1316 . See text for details. The most important observations are: there are clusters bluer than $C-R=1.1$, clusters in the range $1.1-1.3$ show a symmetry with a shifted major axis, clusters of intermediate color define mostly the bulge, but there is also an overdensity of clusters related to outer morphological features to the south.

Interval $1.9<C-R<2.2$.

One would expect old, metal-rich, and reddened clusters in this interval. However, NGC 1316 is no longer visible.

\subsection{Density profiles in various color intervals}

We now consider the radial distributions in different color intervals. This is shown in Figs. 10 and 11. Figure 10 plots the surface densities linearly and includes the color interval $0.8<$ $C-R<1.1$ where the clusters should be younger than $1 \mathrm{Gyr}$. This population is well visible in Fig. 10 at radii corresponding to the bulge region, and less conspicous at larger radii (because of the poor statistics we chose a larger bin in Fig. 11).

In Fig. 11, the left panel shows a blue sample $(0.9<C-R<$ 1.3), the middle panel an intermediate sample $(1.3<C-R<$ $1.6)$, and the right panel $(1.6<C-R<1.9)$ the red sample. It becomes clear that the inflection point in Fig. 8 is caused by the intermediate sample whose density shows a rapid decline at $4.0^{\prime}$ and remains more or less constant for larger radii. Because we are azimuthally averaging along structures that are azimuthally strongly inhomogeneous, this constancy does not reflect a true radial constancy. Interestingly, the inflection point is not visible in the luminosity profile of NGC 1316. Given all evidence, we identify this sample with the bulk of intermediate-age clusters. For the blue and the red sample, the indicated power-law indices refer to the radial interval $0.4<\log (r)<0.9$. The red GC candidates show a somewhat steeper decline. They perhaps preserved their initially more concentrated profile.

Figure 9 shows that the distribution of the intermediate sample at larger radii is concentrated towards the south, roughly delineating Schweizer's L1-structure.

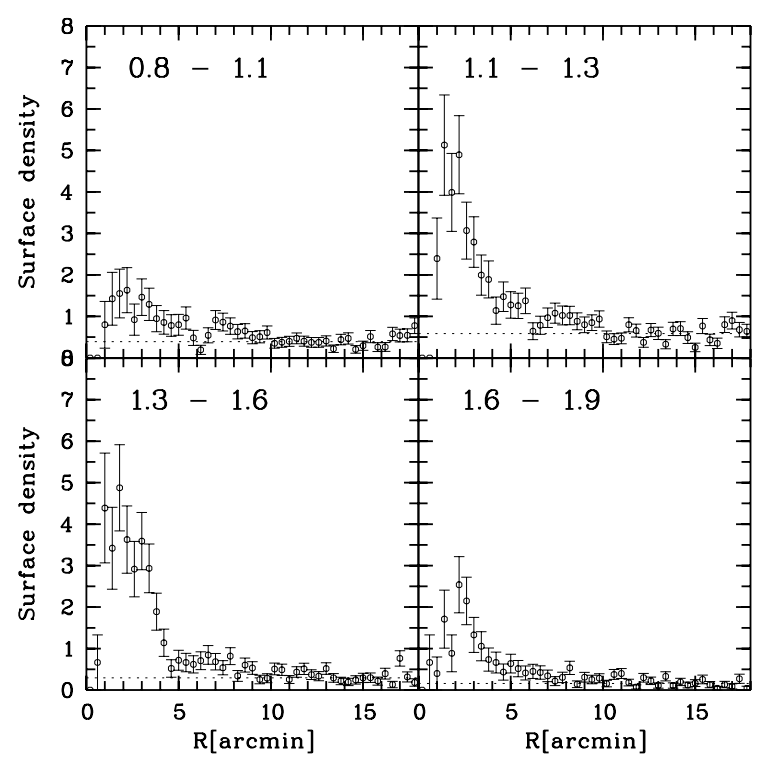

Fig. 10. Background-subtracted surface densities for selected point sources in different $C-R$ color intervals displayed linearly. See Sect. 5.2 for the significance of these intervals and Fig. 11 for a logarithmic display. Although only a poor population, clusters bluer than 1.1 are statistically well visible.

\section{Azimuthal distribution}

Figure 12 shows the azimuthal distribution for three colorsubsamples of GC candidates for the radial range $2^{\prime}-4^{\prime}$ (inner sample) and $4^{\prime}-10^{\prime}$ (outer sample). The bin width is $30^{\circ}$ and 

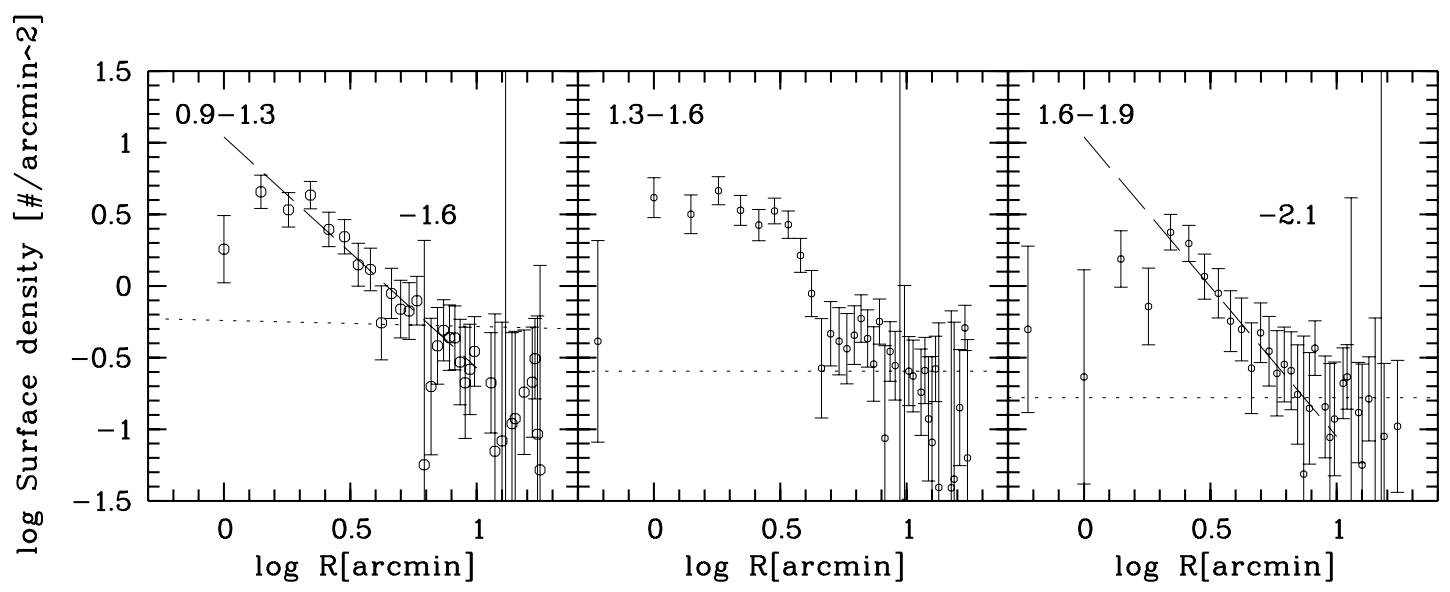

Fig. 11. Surface densities in double-logarithmic display for point sources with the background subtracted and for three different $C-R$ color intervals: 0.9-1.3 (left panel), 1.3-1.6 (middle panel),1.6-1.9 (right panel). For the blue and the red samples, the power-law indices, valid for the radial range between $\log (r)=0.4$ and $\log (r)=0.9$ are indicated. There is no uniform power-law for the intermediate sample. The dashed horizontal lines indicate the backgrounds. The vertical line is an excessively large uncertainty in one bin. Evidently, the inflection point in Fig. 8 is caused by the intermediate sample, which sharply falls off at a distance of $4.5^{\prime}$. We identify this sample with the bulk of intermediate-age clusters.

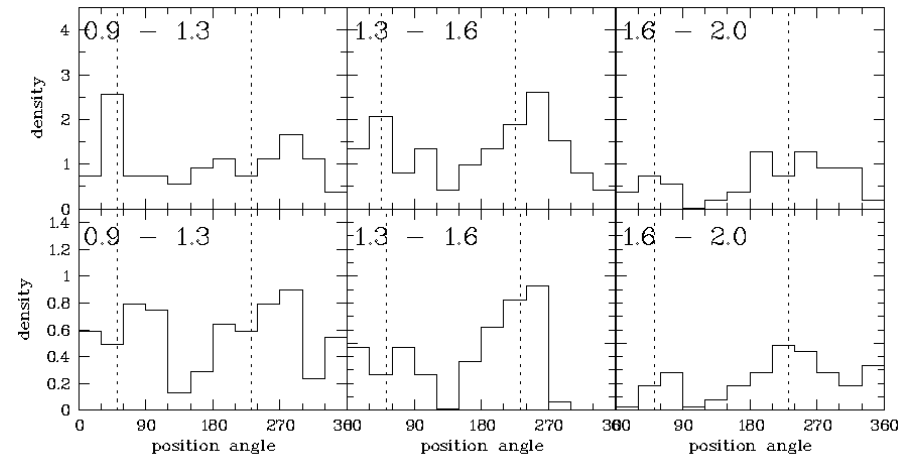

Fig. 12. Distribution of position angles in the radial ranges $2^{\prime}-4^{\prime}$ (upper row) and $4^{\prime}-10^{\prime}$ (lower row) for three color intervals. The major axis is indicated by the vertical dotted lines. Sphericity is detected nowhere.

the counts were performed in circular annuli. The abscissas are the position angles measured counterclockwise with zero in the north. The ordinates are background-subtracted number densities per $\operatorname{arcmin}^{2}$. The first observation is that no subsample shows a spherical distribution, This somewhat contradicts Gómez et al. (2001), who found for their blue sample a spherical distribution (using BRI colors), while their red sample followed the galaxy's ellipticity. We suspect that this is due to our more precise background subtraction and, because of the $C$ filter inclusion, a more precise color subsampling. The highest degree of symmetry is found for the inner intermediate-color sample, once more demonstrating the strong confinement of this population to the bulge. The outer intermediate sample shows the strong dominance of the southern region. Most of these clusters might be attributed to the L1 feature. The red sample, in which we expect old metal-rich clusters, also shows the bulge symmetry.

\section{Luminosity function}

The luminosity function (LF) of the GCS has been derived to very faint magnitudes by HST (Goudfrooij et al. 2001b), although only for the central parts. The main result is that the LF misses a well-defined turn-over magnitude (TOM), which for "normal" elliptical galaxies is an excellent distance indicator

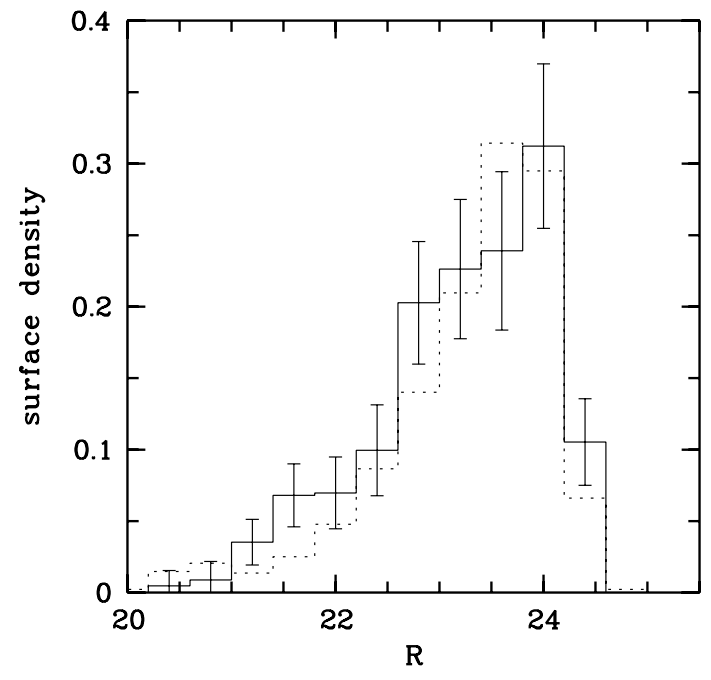

Fig. 13. Luminosity function in $R$ inside a circular annulus between $4^{\prime}$ and $9^{\prime}$ radius. The surface density is counted in numbers/arcmin**2. The background counts were made outside $12^{\prime}$. We do not see the expected turn-over at $R \approx 23.3$. One concludes that also outside the main body of NGC 1316 younger clusters dominate the LF.

(Villegas et al. 2010; Richtler 2003). This can be understood by the relative youth of the cluster system, which still contains unevolved clusters, an effect already noted by Richtler (2003) in galaxies with younger populations.

Our data cannot compete in depth with the HST observations, but we can probe the outer parts of the GCS, where our data are complete down to $R=24 \mathrm{mag}$, to see whether a TOM is visible. With a distance modulus of 31.25 and a TOM of $M_{V}=-7.4$ we expect it at about $V=23.8$ and with a mean $V-R=0.5$. At $R=23.3$, a TOM-brightness outside the main body of NGC 1316 should be clearly visible.

As the radial interval we chose 4-9 arcmin to avoid the galaxy light, have reasonable number counts and avoid the very outskirts. Figure 13 shows the background-corrected LF in this radial range, where the background counts refer to the complete field outside $12^{\prime}$. Surface densities are numbers/arcmin ${ }^{2}$. The 


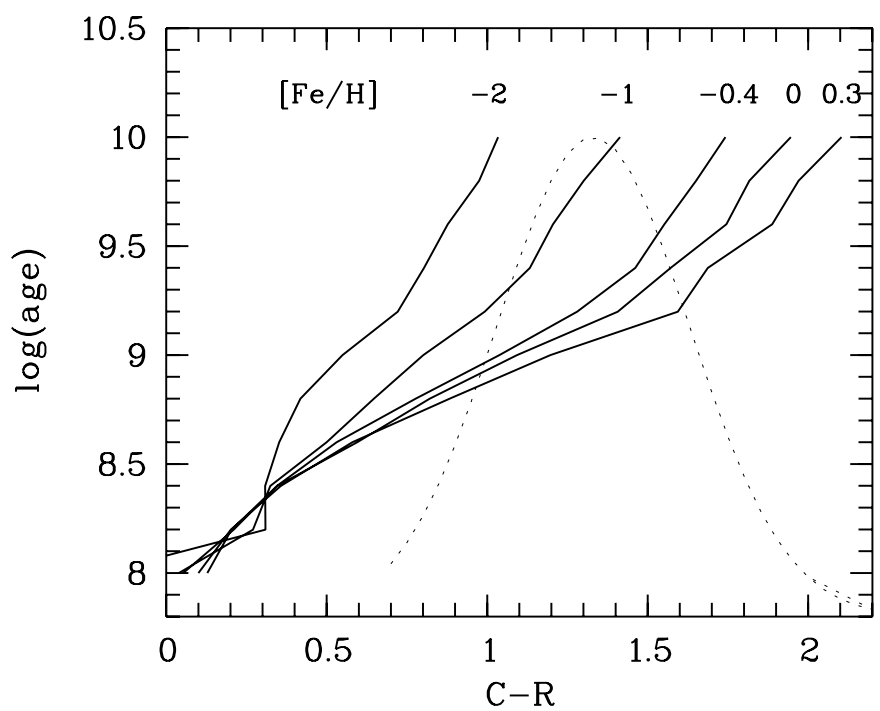

Fig. 14. Theoretical single stellar population models from Marigo et al. (2008) for five different metallicities. Overplotted (in arbitrary units) is a Gaussian representing the color distribution for GC candidates between 2 and 10 arcmin radius. If the cluster sample within $1.3<C-R<$ 1.6 is dominated by objects with at least solar metallicity, many clusters are younger than $2 \mathrm{Gyr}$.

uncertainties are calculated as the square root of backgroundcorrected counts. The dotted histogram shows the background counts.

The expected turn-over at $R \approx 23.3$ mag is not visible, resembling the HST results of Goudfrooij et al. (2004) for the central part and for the red clusters. The conclusion is that in this outer region the LF of intermediate-age clusters contribute significantly. The data of Goudfrooij et al. (2004) indicate a turnover for their blue sample, but see our remarks in Sect. 8.3 for counterarguments.

\section{Discussion}

\subsection{External and internal reddening}

The foreground reddening toward NGC 1316 is low. The allsky maps of Burstein \& Heiles (1982) and (Schlegel et al. 1998) give $E(B-V)=0$ and $E(B-V)=0.02$, respectively. This difference is the zero-point difference between the two reddening laws. According to Schlegel et al., the accuracy of both values is comparable below a reddening of $E(B-V)=0.1$. Therefore the reddening in $C-R$ is $E(C-R)=0.04$ or probably lower, comparable with the uncertainty of the photometric calibration. We therefore preferred not to correct for foreground reddening, also because no conclusion depends on such a precision. The reddening by dust within NGC 1316 outside a radius of $1^{\prime}$ seems to be low as well. We point out that our reddest GC candidates have $C-R \approx 1.9$, which is the appropriate color for old, metal-rich GCs.

\subsection{Theoretical Washington colors}

We used theoretical isochrones to relate cluster ages to Washington colors. Figure 14 compares the color distribution of GC candidates with theoretical models of single stellar populations from Marigo et al. (2008), using their web-based tool (http://stev.oapd.inaf.it/cgi-bin/cmd). Models are shown for five different metallicities. The highest metallicity refers to the stellar population (e.g. Kuntschner 2000) Overplotted (in arbitrary units) is a Gaussian representing the color distribution for $\mathrm{GC}$ candidates between $2^{\prime}$ and $10^{\prime}$ radius.

\subsection{Color distribution and ages}

What are the implications of the color distribution in Fig. 7 for possible scenarios of the cluster formation history of NGC 1316? First we compare our $C-R$ photometry with the HST-photometry in $B, V, I$ of the cluster sample of Goudfrooij et al. (2001a). These authors found two peaks at $B-I=1.5$ and $B-I=1.8$. Translating these values into Washington colors with the help of Fig. 1, one estimates for these peaks $C-R=1.1$ and $C-R=1.4$, respectively. This is indeed consistent with what is seen in Fig. 7. Already Goudfrooij et al. (2001b) interpreted the peak at $B-I=1.8$ as the signature of intermediate-age clusters with ages around $3 \mathrm{Gyr}$, which was backed up by the spectroscopic ages and metallicities derived for a few bright objects (Goudfrooij et al. 2001a). From Fig. 14 one would rather assign an age of somewhat less than 2 Gyr. If the bluer peak indicates old, metal-poor clusters of the pre-merger components, the metallicity distribution of old clusters must be strongly biased to very metal-poor objects and thus be very different from that in "normal" globular cluster systems of ellipticals (and also spirals), which show a more or less universal peak at $C-R=1.35$ (Bassino et al. 2006b).

Since we can identify clusters with ages even younger than what would correspond to $C-R=1.1$, that means below $1 \mathrm{Gyr}$, one rather expects a mixture of old and intermediate-age clusters of unknown proportions. The hypothesis is interesting that the blue peak does not represent an overabundance of very metalpoor clusters, but marks a second burst of star formation with an age of about 1 Gyr. A counterargument could be that the luminosity function in a $V, V-I$ diagram seems to show a turn-over at about $V=24.6 \mathrm{mag}$ (Goudfrooij et al. 2004). However, several points related to Fig. 3 of Goudfrooij et al. create doubts whether this turn-over really corresponds to the universal turnover magnitude in GCS. Firstly, Goudfrooij et al. (2004) adopted a distance of $22.9 \mathrm{Mpc}$, which means a brightening of $0.5 \mathrm{mag}$ in absolute magnitudes with respect to the supernova distance of $17.8 \mathrm{Mpc}$, we adopted. Accordingly, the turn-over would be at $M_{V}=-6.7$, i.e. almost $0.7 \mathrm{mag}$ fainter than the "normal" turn-over, while one would expect the turn-over to be brighter due to the bias to low metallicities. Secondly, the turn-over itself is not well defined, the highest number count being even fainter than the formal turn-over from the fit, which leaves the question open whether there is a decline at fainter magnitudes or not. Thirdly, their color interval, which defines "old clusters", is $0.55<V-I<0.97$, a sample which clearly includes intermediate-age clusters (here we also point to the superiority of the Washington system with respect to $V-I$ and refer the reader to Fig. 7 of Dirsch et al. 2003b).

Evidence from our photometry comes from the fact that the red peak is strongly constrained to the bulge (Figs. 11 and 10). The blue clusters, on the other hand, are abundant still in the outskirts of NGC 1316. If this outer cluster population would consist exclusively of old clusters, then a turn-over magnitude should be visible in our luminosity function, which is not even corrected for incompleteness.

The absence of the striking red peak in the outer region shows that these stellar populations do not stem from the first star-burst. The dynamical age of these regions is unknown, but plausibly younger than the inner star burst. 
The light of the inner bulge is redder than the peak color of the GCS by about $0.15 \mathrm{mag}$ in $C-R$ and shows only a slight radial transition to bluer colors ${ }^{1}$.

A differential reddening between clusters and stellar population is not probable. The selection of cluster candidates between $1^{\prime}$ and $3^{\prime}$ (236 objects), where the reddening should be strongest, still gives a peak color of $C-R=1.38$, while the galaxy has $C-R=1.57$. An explanation for this difference may be sought in the fact that the clusters are (more or less) single stellar populations of intermediate-age, while the total bulge light has a contribution from old populations. We argue that this contribution is significant.

We find statistical evidence for clusters between $0.8<C-$ $R<1.1$ which are neither old and metal-poor nor can be related to a starburst 2 Gyr ago, but must be younger even if their metallicities are lower than solar. Plausibly, these clusters should have a metallicity at least as high as the intermediate-age stellar population, if they formed in NGC 1316.

However, there is still the possibility that infalling dwarf galaxies provided clusters with low metallicities

\subsection{Comparison with spectroscopic ages}

Goudfrooij et al. (2001b) provided spectroscopic ages and metallicities for three clusters, their objects 103, 114, and 210. There exist $C-R$ measurements for 103 and 210 (114 is too close to the center). These clusters have solar abundance and a common age (within the uncertainties) of $3 \mathrm{Gyr}$. The $C-R$ colors for 103 and 210 are 1.48 and 1.51 , respectively. A comparison with Fig. 14 shows that these colors are slightly too blue for full consistency with the model colors for $3 \mathrm{Gyr}$ and solar abundance. A shift to the red of $0.1 \mathrm{mag}$ in $C-R$ would be necessary to achieve perfect agreement, but given that various models with their respective uncertainties and the uncertainty of the absolute photometric calibration are involved, one can consider the agreement to be satisfactory. In any case, these two clusters do not seem to be reddened.

\subsection{Subpopulations}

A bimodal color distribution is not exclusively a feature of elliptical galaxies, as the example of the Milky Way shows. As a working hypothesis, we assumed that the color histogram of old GCs of NGC 1316 also has a bimodal appearance. Moreover, we assumed that clusters redder than the galaxy color belong to the old, metal-rich subpopulation. Bassino et al. (2006b) compiled values for the blue and the red peak of early-type galaxies in the Washington system. Adopting these values, we fixed the Gaussian of the red peak with a maximum at $C-R=1.75$ and a dispersion of 0.15 . We cannot distinguish old, metal-poor clusters from younger, metal-rich clusters that populate the same color range, but may assume that their color distribution follows the almost universal distribution known from other galaxies, for which Bassino et al. (2006b) quotes $C-R=1.32$ for the peak color and $0.15 \mathrm{mag}$ for the dispersion.

To get an impression of what the pre-merger GCSs could look like, we fitted the corresponding Gaussian to the color bins

\footnotetext{
1 Strictly speaking, this difference is not exactly valid, because we measured the color of the stellar population by using the total projected light and calculated a color for the GCs by averaging over magnitudes. The difference, however, is negligible.
}

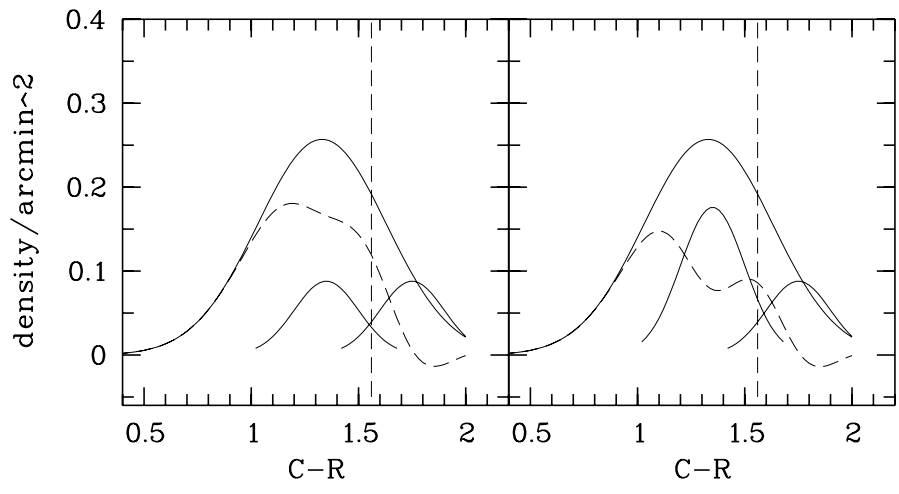

Fig. 15. In both panels, the broad Gaussian (solid line) represents the color distribution of the entire GCS. The two narrower Gaussians represent the assumed bimodality of old metal-poor and metal-rich clusters. The dashed line results from subtracting the bimodal color distribution. In the left panel (case 1), intermediate-age clusters dominate, in the right panel (case 2), old clusters dominate. Case 2 is less consistent with the properties of the GC population.

redder than $C-R=1.6$, assuming that all clusters redder than the galaxy light are old. The amount of old, metal-poor clusters is unknown, but we assumed two cases: equal to and twice the metal-rich population. The subtraction of these two Gaussians from the Gaussian describing the entire sample down to $R=$ 24 mag in Sect. 4 reveals the GC population, which has been formed in the merger and and perhaps in following star-forming events.

Figure 15 shows these two cases. In the left panel (case 1) the dashed line results from the subtraction of the assumed bimodal color distribution of old clusters. In this case, intermediate-age clusters are dominating the color interval 1.3-1.6. In the the right panel (case 2), old clusters dominate, which contradicts the behaviour of clusters in this color range. Therefore, case 1 may be closer to the true situation.

\subsection{A star formation history}

The star formation history of NGC 1316 was probably complex. A merger event is not a simple infall, but is preceded by close encounters, which can trigger starbursts, in time separated by the orbital period. See, for example, Teyssier et al. (2010) and Di Matteo et al. (2008) for modern simulations of mergers and starbursts. Teyssier et al. resolved the multi-phase ISM and found that the main trigger for starbursts is not a large-scale gas flow, but a fragmentation of gas into cold and dense clumps, favoring star cluster formation. A realistic scenario would probably involve many individual starbursts, which can perhaps be identified in future high $\mathrm{S} / \mathrm{N}$ spectra of globular clusters in NGC 1316. Our intention in simplifying the star formation history is to show that to bring photometric properties and dynamical determined mass-to-light ratios into agreement, the properties of the pre-merger population rule out an elliptical galaxy. Moreover, a contribution of a population significantly younger than 2 Gyr is needed.

We therefore ask whether it is possible to find a simple model, inspired by the color distribution of GCs, which is consistent with the color and the mass-to-light ratio of the bulge stellar population. We adopted three populations: a pre-merger population (pop. 1) with an a-priori unknown age, and two populations representing starbursts with ages 2 Gyr (pop. 2) and $0.8 \mathrm{Gyr}$ 
(pop. 3). Since the luminosity-weighted metallicity of the bulge is at least solar (Kuntschner 2000), we adopted solar metallicity for each of them. The color of NGC 1316 then results from a composite of these populations, to which we assign the integrated color $C-R=1.55$ (see Fig. A.5).

Mass-to-light ratios for the inner bulge have been quoted by several authors in various photometric bands. To transform an $M / L$-value from one band to another, is not completely trivial for NGC 1316, since published aperture photometries (Prugniel \& Heraudeau 1998) measured a color that is reddened by the dust within about $1^{\prime}$ radius. We therefore adopted theoretical colors (including Washington colors) from the Padova models (http://stev.oapd.inaf.it/cgi-bin/cmd; Marigo et al. 2008). The relation between $M / L$-ratios (denoted by $\Gamma$ ) in filters $i$ and $j$ is

$$
\frac{\Gamma_{i}}{\Gamma_{j}}=\frac{L_{j}}{L_{i}} \frac{L_{i, \odot}}{L_{j, \odot}} .
$$

$C-R=1.55$ corresponds to a good approximation to a single stellar population with solar metallicity and an age of $2.5 \mathrm{Gyr}$. For that population we found $B-V=0.81, B-R=1.34$, and $R-I=0.56$. Furthermore, we adopt the solar absolute magnitudes of (Binney \& Merrifield 1998, p. 53) and accordingly were able to transform $M / L$-values into the $R$-band.

Schweizer (1981) gave $M / L_{V}=1.3 \pm 0.2$ for a distance of $32.7 \mathrm{Mpc}$, corresponding to $M / L_{R}=2.1$ for our distance $(M / L$ is inversely proportional to the distance). Shaya et al. (1996) gave $M / L_{V} \approx 2.2 \pm 0.2$, corresponding to $M / L_{R} \approx 1.9$ (distance $16.9 \mathrm{Mpc}$ ). Arnaboldi et al. (1998), by using dynamics of planetary nebulae, quoted $M / L_{B} \approx 7.7$ inside a radius of $200^{\prime \prime}$ (distance $16.9 \mathrm{Mpc}$ ). However, they found lower values for smaller radii, down to $M / L_{B} \approx 4.3$ at a radius of $45^{\prime \prime}$ which corresponds to $M / L_{R} \approx 3.1$. This early result, based on only a few planetary nebulae, is superseded by the recent work of McNeil-Moylan et al. (2012), who quoted $M / L_{B}=2.8$ (distance $21.5 \mathrm{Mpc}$ ) for the stellar population, which transforms into $M / L_{R} \approx 2.6$. Owing to the large database of their dynamical model, this value certainly has a high weight. However, it is based on spherical modeling and the authors caution that it may change with more realistic models. Nowak et al. (2008), modeling the central kinematics with a black hole, have found for the central region a value in the $K_{\mathrm{s}}$-band between 0.7 and 0.8 (distance $18.6 \mathrm{Mpc}$ ), corresponding to a value between 2.8 and 3.2 in the $R$-band (setting $M_{K} s / M_{K \mathrm{~s}, \odot}=M_{K} / M_{K, \odot}$ and adopting $R-K=2.60$ for the stellar population). Future work will probably lower the uncertainties. For the moment, $M / L_{R} \approx 2.5$ seems to be a good value to adopt.

Perhaps the best empirical determinations of $M / L$-values of stellar populations of early-type galaxies come from the SAURON-group (Cappellari et al. 2006). The highest values that they quote in the $I$-band inside one effective radius, are around 5 (their "Jeans"-values). Considering that a dark halo can contribute up to $8 \%$ of the total mass, values around 4 for metalrich, old populations seem reasonable. The Padova models give $M / L_{I}=4.1$ for a 12 Gyr old population of solar metallicity (with $\left.M_{I, \odot}=+4.10\right)$, assuming a log-normal IMF (Chabrier 2001), reproducing the empirical values quite well.

Adopting their $M / L$-ratios also for younger populations, we now can assign colors and $M / L$-values to our model populations and estimate the properties of the pre-merger under simplifying assumptions.

Table 3 lists the models, ages, metallicities, Washington colors, and $R$-band mass-to-light-ratios (Cols. 1-5). We assumed two different pre-merger populations with ages of
Table 3. Properties of three adopted single stellar populations and their composite.

\begin{tabular}{lcccccc}
\hline \hline $\begin{array}{l}\text { Pop. } \\
\text { (1) }\end{array}$ & $Z$ & Age[Gyr] & $C-R$ & $M / L_{R}$ & $L_{R}$ & $M\left[M_{\odot}\right]$ \\
\hline $1 \mathrm{a}$ & 0.019 & 12 & 1.99 & 5.2 & 0.60 & $3.18 \times 10^{11}$ \\
2 & 0.019 & 2 & 1.49 & 1.1 & 0.13 & $1.55 \times 10^{10}$ \\
3 & 0.019 & 0.8 & 0.96 & 0.57 & 0.27 & $1.53 \times 10^{10}$ \\
\hline Composite & & & 1.55 & 3.4 & 1 & $3.48 \times 10^{11}$ \\
\hline $1 \mathrm{~b}$ & 0.019 & 7 & 1.84 & 3.4 & 0.67 & $2.36 \times 10^{11}$ \\
2 & 0.019 & 2 & 1.49 & 1.1 & 0.11 & $1.22 \times 10^{10}$ \\
3 & 0.019 & 0.8 & 0.96 & 0.57 & 0.22 & $1.26 \times 10^{10}$ \\
\hline Composite & & & 1.55 & 2.55 & 1 & $2.60 \times 10^{11}$ \\
\hline
\end{tabular}

12 Gyr (pop. 1a) and 7 Gyr (pop. 1b). The equation to be solved is then

$$
(C-R)_{\text {integrated }}=1.55=-2.5 \log \left(\frac{(L 1+L 2+L 3)_{C}}{(L 1+L 2+L 3)_{R}}\right),
$$

where $\mathrm{L} 1, \mathrm{~L} 2, \mathrm{~L} 3$ are the respective luminosities in $C$ and $R$. If we use the $R$-luminosity of the bulge as unit and assume a certain mass proportion of pop. 2 and pop. 3, one can solve for L2 and L3. Since in case 1 of Fig. 15 the young clusters are of comparable number, we assumed pop. 2 and pop. 3 to contribute with equal stellar mass. L2 and L3 ( $R$-band) are given in Col. 6 . Column 7 gives the resulting stellar masses for the bulge (inside a radius of $240^{\prime \prime}$ ) using the bulge luminosity of Table A.1. These values are upper limits because in reality one must use the deprojected bulge luminosities, but the global symmetry of NGC 1316 is not known.

With these values, one can calculate the resulting $M / L$ of the composite population.

What one learns from this exercise is that a pre-merger population of 12 Gyr with solar metallicity, representative of an elliptical galaxy, does not agree well with a composite $M / L_{R}=2.5$. In contrast, a 7-Gyr old population fits well, which one would expect if the pre-merger was one or two spiral galaxies with an already existing mix of old to intermediate-age populations.

Moreover, a population significantly younger than $2 \mathrm{Gyr}$ is necessary for $M / L_{R}=2.5$. If only the $2 \mathrm{Gyr}$ population would exist, it would be so dominant $\left(L 2_{R}=0.85\right.$ for a 12 Gyr premerger population) that $M / L_{R}=1.7$. This value would even be lower for a younger pre-merger population.

These considerations are valid only for the bulge. In the outer parts, the galaxy becomes bluer, but we have no knowledge of the metallicities or ages. However, one might suspect that younger ages dominate over decreasing metallicity, because the southern L1-structure is traced by GCs of probably intermediate age.

We therefore have a total stellar bulge mass of about $2.6 \times$ $10^{11} M_{\odot}$, of which only $10 \%$ have been produced in both starbursts. These $10 \%$ are, however, responsible for the majority of bright (!) GCs, emphasizing once more that the efficiency of GC formation in phases of high star formation rates is greatly enhanced (Whitmore \& Schweizer 1995; Larsen \& Richtler 2000; de Grijs et al. 2003b,a; Kravtsov \& Gnedin 2005).

One has to account for a gas mass of $2.6 \times 10^{10} M_{\odot}$, which has been transformed into stars. Since we must assume a strong galactic wind with heavy mass loss during the burst phases, the total gas mass needed was probably higher. A gas mass of 
this order is easily provided by one or two spiral galaxies (e.g. McGaugh \& de Blok 1997).

\section{Summary and concluding remarks}

We presented wide-field photometry $\left(36 \times 36 \operatorname{arcmin}^{2}\right)$ in Washington $C$ and Kron-Cousins $R$ around the merger remnant NGC 1316 (Fornax A) to investigate the globular cluster system of NGC 1316 on a larger scale than has been done before. The data consist of MOSAIC images obtained with the 4-m Blanco telescope at CTIO. The Washington system is particularly interesting because of its good metallicity resolution in GCSs containing old clusters and for the comparison with the well-established bimodal color distribution of elliptical galaxies.

Our main findings are:

The GC candidates are well confined to the region of the optical extension of NGC 1316. Outside a radius of 12' (corresponding to $62 \mathrm{kpc}$ ), a cluster population is statistically not detectable.

The color distribution of GC candidates is quite different from that of old elliptical galaxies. The entire system down to $R=24$ mag (which we estimate to be the completeness limit) shows a broad Gaussian-like color distribution with a peak at $C-R=1.37$, somewhat bluer than the color of the inner bulge. A selection of brighter GC candidates shows striking color peaks at $C-R=1.1$ and $C-R=1.4$ for bulge objects. The color distribution of objects at radii larger than $4^{\prime}$ is unimodal with a peak about $C-R=1.2$, bluer than expected for an old GC population. This is a hint that younger GCs also exist outside the bulge. We found a small population of GC candidates bluer than $C-R=1.0$, which is the limit for old metal-poor clusters. Clusters bluer than $C-R=0.8$ are statistically not detectable, but casual confirmations through radial velocities show that there are sporadically clusters as blue as $C-R=0.4$.

The intermediate color interval $1.3<C-R<1.6$ shows a radial surface density profile very different from the other color regimes. These objects are strongly confined to the inner bulge, showing an inflection at about $4^{\prime}$. For radii larger than $4^{\prime}$, their distribution is azimuthally inhomogeneous with a pronounced concentration in the area of Schweizer's (1980) L1-structure. The blue clusters $(0.9<C-R<1.3)$ fall off without showing an inflection with a power-law exponent of -1.6 . The red clusters $(1.6<C-R<2)$ follow a somewhat steeper power-law with an exponent of -2.1 .

Outside the bulge, the luminosity function of cluster candidates does not show the turn-over expected for an old cluster system, supporting the conclusion from the color distribution that younger clusters contribute significantly also at larger radii.

Assuming that younger clusters have at least the metallicity of the bulge population, one can assign ages, using theoretical Washington isochrones and integrated colors. We used the models of Marigo et al. 2008. The peak at $C-R=1.4$ then corresponds to an age of about $1.8 \mathrm{Gyr}$, the peak at $C-R=1.1$ to an age of $0.8 \mathrm{Gyr}$, and the bluest colors to less than $0.5 \mathrm{Gyr}$. The brighter population of cluster candidates therefore consists of intermediate-age clusters, while older clusters of the pre-merger galaxy are progressively mixed in at fainter magnitudes.

The peaks probably stem from epochs of very high star formation rates, connected to one or several merger events. The bluest clusters indicate ongoing star formation with ages younger than $0.8 \mathrm{Gyr}$.

Guided by the color distribution of GCs, we presented a simple model, which as the main ingredient adopts two burst of star formation with ages 2 Gyr and 0.8 Gyr added to an older population of solar metallicity. To reproduce the color of the bulge of NGC 1316, and the stellar $M / L$-value from dynamical estimates, this older population has an age of about $7 \mathrm{Gyr}$ as a single stellar population. In reality, this population is expected to be a mix of populations and therefore the age indicates spiral galaxies as merger components rather than an old elliptical galaxy.

The stellar masses of the younger populations account for only $10 \%$ of the total stellar mass, while their luminosity contributes $30 \%$ of the total luminosity.

The general picture of NGC 1316 as a merger remnant with a complex mix of stellar populations is reflected in the properties of its globular cluster system. Because our GC colors are degenerate regarding ages and abundances, future spectroscopic studies may refine some of the present findings, but the global scenario of a primary starburst about 2 Gyr ago with a long-lasting aftermath of star formation, probably discontinuous in time, seems to be well established. However, the exact nature of the merger remains to be investigated. Was there one dominant spiral galaxy that merged with gas-rich dwarf galaxies or a spiral-spiral merger? What is the nature of the dark halo? In answering these questions, globular clusters will play an important role.

Acknowledgements. We thank the anonymous referee for valuable remarks and constructive criticism. T.R. acknowledges financial support from the Chilean Center for Astrophysics, FONDAP Nr. 15010003, from FONDECYT project Nr. 1100620, and from the BASAL Centro de Astrofisica y Tecnologias Afines (CATA) PFB-06/2007. He also thanks the Aryabhatta Research Institute of Observational Sciences, Nainital, for warm hospitality and financial support. LPB gratefully acknowledges support by grants from Consejo Nacional de Investigaciones Científicas y Técnicas and Universidad Nacional de La Plata (Argentina). We thank Richard Lane for a careful reading of a draft version.

\section{References}

Arnaboldi, M., Freeman, K. C., Gerhard, O., et al. 1998, ApJ, 507, 759

Bassino, L. P., Faifer, F. R., Forte, J. C., et al. 2006a, A\&A, 451, 789

Bassino, L. P., Richtler, T., \& Dirsch, B. 2006b, MNRAS, 367, 156

Bedregal, A. G., Aragón-Salamanca, A., Merrifield, M. R., \& Milvang-Jensen, B. 2006, MNRAS, 371, 1912

Binney, J., \& Merrifield, M. 1998, Galactic Astronomy (Princeton University Press)

Blakeslee, J. P., Jordán, A., Mei, S., et al. 2009, ApJ, 694, 556

Brodie, J. P., \& Strader, J. 2006, ARA\&A, 44, 193

Burstein, D., \& Heiles, C. 1982, AJ, 87, 1165

Cappellari, M., Bacon, R., Bureau, M., et al. 2006, MNRAS, 366, 1126

Chabrier, G. 2001, ApJ, 554, 1274

Chernin, A. D. 1998, Astrophys., 41, 399

Davies, B., Bastian, N., Gieles, M., et al. 2011, MNRAS, 411, 1386

de Grijs, R., Anders, P., Bastian, N., et al. 2003a, MNRAS, 343, 1285

de Grijs, R., Lee, J. T., Mora Herrera, M. C., Fritze-v. Alvensleben, U., \& Anders, P. 2003b, New Astron., 8, 155

Di Matteo, P., Bournaud, F., Martig, M., et al. 2008, A\&A, 492, 31

Dirsch, B., Richtler, T., \& Bassino, L. P. 2003a, A\&A, 408, 929

Dirsch, B., Richtler, T., Geisler, D., et al. 2003b, AJ, 125, 1908

Dirsch, B., Schuberth, Y., \& Richtler, T. 2005, A\&A, 433, 43

Dobbs, C. L., Theis, C., Pringle, J. E., \& Bate, M. R. 2010, MNRAS, 403, 625

Fabbiano, G., Kim, D.-W., \& Trinchieri, G. 1992, ApJS, 80, 531

Feigelson, E. D., Laurent-Muehleisen, S. A., Kollgaard, R. I., \& Fomalont, E. B. 1995, ApJ, 449, L149

Fukazawa, Y., Botoya-Nonesa, J. G., Pu, J., Ohto, A., \& Kawano, N. 2006, ApJ, 636, 698

Geisler, D. 1996, AJ, 111, 480

Gómez, M., Richtler, T., Infante, L., \& Drenkhahn, G. 2001, A\&A, 371, 875

Goudfrooij, P., Alonso, M. V., Maraston, C., \& Minniti, D. 2001a, MNRAS, 328, 237

Goudfrooij, P., Mack, J., Kissler-Patig, M., Meylan, G., \& Minniti, D. 2001b, MNRAS, 322, 643

Goudfrooij, P., Gilmore, D., Whitmore, B. C., \& Schweizer, F. 2004, ApJ, 613, L121

Graham, J. A. 1987, BAAS, 19, 1048 
Graham, J. A., \& Fassett, C. I. 2002, ApJ, 575, 712

Grillmair, C. J., Forbes, D. A., Brodie, J. P., \& Elson, R. A. W. 1999, AJ, 117, 167

Harris, W. E. 2010, Royal Society of London Philosophical Transactions Series A, 368, 889

Harris, H. C., \& Canterna, R. 1977, AJ, 82, 798

Horellou, C., Black, J. H., van Gorkom, J. H., et al. 2001, A\&A, 376, 837

Iyomoto, N., Makishima, K., Tashiro, M., et al. 1998, ApJ, 503, L31

Kaneda, H., Onaka, T., \& Sakon, I. 2007, ApJ, 666, L21

Kim, D.-W., \& Fabbiano, G. 2003, ApJ, 586, 826

Kim, D.-W., Fabbiano, G., \& Mackie, G. 1998, ApJ, 497, 699

Konami, S., Matsushita, K., Nagino, R., et al. 2010, PASJ, 62, 1435

Kravtsov, A. V., \& Gnedin, O. Y. 2005, ApJ, 623, 650

Kundu, A., \& Whitmore, B. C. 2001, AJ, 121, 2950

Kuntschner, H. 2000, MNRAS, 315, 184

Lanz, L., Jones, C., Forman, W. R., et al. 2010, ApJ, 721, 1702

Larsen, S. S., \& Richtler, T. 1999, A\&A, 345, 59

Larsen, S. S., \& Richtler, T. 2000, A\&A, 354, 836

Larsen, S. S., Brodie, J. P., Huchra, J. P., Forbes, D. A., \& Grillmair, C. J. 2001, AJ, 121, 2974

Mackie, G., \& Fabbiano, G. 1998, AJ, 115, 514

Marigo, P., Girardi, L., Bressan, A., et al. 2008, A\&A, 482, 883

McGaugh, S. S., \& de Blok, W. J. G. 1997, ApJ, 481, 689

McNeil-Moylan, E. K., Freeman, K. C., Arnaboldi, M., \& Gerhard, O. E. 2012, A\&A, 539, A11

Mieske, S., Hilker, M., Jordán, A., et al. 2008, A\&A, 487, 921

Nagino, R., \& Matsushita, K. 2009, A\&A, 501, 157

Nowak, N., Saglia, R. P., Thomas, J., et al. 2008, MNRAS, 391, 1629
Peng, E. W., Ford, H. C., \& Freeman, K. C. 2004a, ApJS, 150, 367 Peng, E. W., Ford, H. C., \& Freeman, K. C. 2004b, ApJ, 602, 705 Prugniel, P., \& Heraudeau, P. 1998, A\&AS, 128, 299

Rejkuba, M., Harris, W. E., Greggio, L., \& Harris, G. L. H. 2011, A\&A, 526, A123

Richtler, T. 2003, in Stellar Candles for the Extragalactic Distance Scale, eds. D. Alloin, \& W. Gieren (Berlin: Springer Verlag), Lect. Notes Phys., 635,281

Richtler, T., Salinas, R., Misgeld, I., et al. 2011, A\&A, 531, A119

Saikia, D. J., \& Jamrozy, M. 2009, Bull. Astron. Soc. India, 37, 63

Schlegel, D. J., Finkbeiner, D. P., \& Davis, M. 1998, ApJ, 500, 525

Schuberth, Y., Richtler, T., Hilker, M., et al. 2010, A\&A, 513, A52

Schweizer, F. 1980, ApJ, 237, 303

Schweizer, F. 1981, ApJ, 246, 722

Shaya, E. J., Dowling, D. M., Currie, D. G., et al. 1996, AJ, 111, 2212

Sikkema, G., Peletier, R. F., Carter, D., Valentijn, E. A., \& Balcells, M. 2006, A\&A, 458, 53

Stritzinger, M., Burns, C. R., Phillips, M. M., et al. 2010, AJ, 140, 2036

Temi, P., Mathews, W. G., \& Brighenti, F. 2005, ApJ, 622, 235

Teyssier, R., Chapon, D., \& Bournaud, F. 2010, ApJ, 720, L149

Thomas, D., Maraston, C., Bender, R., \& Mendes de Oliveira, C. 2005, ApJ, 621,673

Tremaine, S., Gebhardt, K., Bender, R., et al. 2002, ApJ, 574, 740

Villegas, D., Jordán, A., Peng, E. W., et al. 2010, ApJ, 717, 603

Whitmore, B. C., \& Schweizer, F. 1995, AJ, 109, 960

Woodley, K. A., Gómez, M., Harris, W. E., Geisler, D., \& Harris, G. L. H. 2010a, AJ, 139, 1871

Woodley, K. A., Harris, W. E., Puzia, T. H., et al. 2010b, ApJ, 708, 1335

Pages 15 to 18 are available in the electronic edition of the journal at http://wWw . aanda.org 
T. Richtler et al.: The globular cluster system of NGC 1316. I.

\section{Appendix A: Morphology of NGC 1316}

The morphology of NGC 1316 has been discussed several times (e.g. Schweizer 1980; Mackie \& Fabbiano 1998), but to our knowledge, a wide-field deep CCD image of NGC 1316 does not exist in the literature. Moreover, the color map of the inner region exhibits details that are worth to be shown.

\section{A.1. Global morphology}

A deep wide-field image of NGC 1316 reveals a wealth of faint structures. We here focus on GCs, but it is appropriate to make a few remarks on the global morphology. Schweizer (1980) gave a thorough morphological description of the NGC 1316 complex on a scale of about 1 degree. For orientation and labels of the various features, we refer the reader to Fig. 2 of Schweizer (1980).

It is interesting to see that our deep CCD-image (Fig. A.1) is not much superior to photographic Schmidt plates regarding the detection of faint areal structures. In Fig. A.1 almost all features labeled by Schweizer are visible. There are only two structures that we do not share. One is the very faint arc to the SE (Schweizer's Fig. 9), which is outside our field. The other is an extremely faint curved arc (resembling a "spiral arm") that appears as a continuation of the L1 structure reaching out to the NW. Regarding this feature, one may think of the "rows" in grand design spiral galaxies where parts of spiral arms are straight lines rather than curved. This has not been discussed much in the literature. Chernin (1998) gave a gas dynamical interpretation, but encounters may also play a role.

For example, the structure of the grand design spiral M 51 has been analyzed by Dobbs et al. (2010) through hydrodynamical models. They identified tidal influences (in the case of M 51 the interaction with NGC 5195) as a main driver for shaping spiral structure. In analogy, NGC 1316 could have interacted with its companion NGC 1317 or with a subsequently merged galaxy.

Intuitively, the region outside the bulge gives the impression of an inclined disk rotating clockwise. Practically all loops are consistent with this sense of rotation except the outermost loop in the SW. However, a bulge component kinematically dominates outside 35" (Bedregal et al. 2006).

Very noticable is the sharp border in the south. This may be another hint that we are looking onto a (thick?) disk since threedimensional structures in projection should not be so sharply confined.

The distribution of GCs is well-confined to the luminous area seen in Fig. A.1. We emphasize the morphological similarity of NGC 1316 with NGC 474/470. Sikkema et al. (2006) presented $V-I$ photometry of its GCS and only found a broad blue peak, similar to our entire sample.

\section{A.2. The surface brightness profile}

Owing to the inner saturated region, we can measure the surface brightness profile of NGC 1316 in the $R$-band on our MOSAIC image only from about $50^{\prime \prime}$ outward. To enable the comparison with published aperture photometry, we used the ellipse-task in IRAF to measure (almost) circular "isophotes" and tried to find an analytic spherical model for the surface brightness. We integrated this radial model and calculated aperture photometric values, which we compared with the compilation of aperture photometries of Prugniel \& Heraudeau (1998). For the model, we chose a "beta-model", which worked surprisingly well. The
Table A.1. Characteristic values of the spherical photometric model.

\begin{tabular}{lcc}
\hline \hline Radius & $M_{R}$ & $L_{R} / L_{\odot}$ \\
\hline $240^{\prime \prime}$ (bulge) & -23.07 & $1.02 \times 10^{11}$ \\
$600^{\prime \prime}$ & -23.33 & $1.3 \times 10^{11}$ \\
\hline
\end{tabular}

exponent of -1 enables deprojection and integrated mass to be written analytically (which here would result in only approximate values, since the system is not spherical, e.g. see Schuberth et al. 2010; Richtler et al. 2011).

$\mu(R)=-2.5 \log \left(a_{1}\left(1+\left(\frac{R}{R_{\mathrm{c}}}\right)^{2}\right)^{\alpha}\right)$

with $a_{1}=3.902 \times 10^{-7}, R_{\mathrm{c}}=8^{\prime \prime}, \alpha=-1.0$. To transform this surface brightness into $L_{\odot} / p c^{2}$, one has to apply a factor $2.56 \times 10^{10}$ to the argument of the logarithm (where we used $M_{R, \odot}=4.45$ ). The foreground extinction in the $R$-band is 0.056 mag (Schlegel et al. 1998), which we neglected.

Figure A.2 shows in its upper panel the beta-model (solid line), the spherical model (open circles), and measurements of elliptical isophotes (ellipticity 0.3 , position angle $50^{\circ}$ ). These measurements were shifted by 0.5 mag to enhance the visibility. In the spherical model, the bulge does not emerge as a separate entity, because the sphericity introduces some smoothing. We compared our model with published aperture photometry (Prugniel \& Heraudeau 1998) by integrating within given apertures. The result is shown in Fig. A.3 for eight apertures and is found to be very satisfactory.

Table A. 1 lists the absolute $R$-magnitudes and corresponding luminosities for the bulge and the total extension of NGC 1316. The effective radius is $69.5^{\prime \prime}$, corresponding to almost exactly $6 \mathrm{kpc}$.

\section{A.3. Color image}

The color image is restricted in size because of the strong flatfield features present on the western part of the C-image.

Figure A.4 displays the color in magnitudes. Its size is $23.3^{\prime} \times 14.5^{\prime}$. After subtracting the sky, we divided the C-image by the $R$-image and converted the resulting image to a magnitude scale. We selected from the GC photometry 10 of the brightest non-saturated objects at small radii and applied their calibrated magnitudes to the galaxy light to make sure that the galaxy colors are measured differentially to the GC colors. The resulting scatter is $0.03 \mathrm{mag}$ in $C-R$. The dynamical range of Fig. A.4 covers the color interval 1.3-1.9, which was found to be the most satisfactory. Dark is blue and bright is red. The magnitude scale itself is displayed logarithmically. Owing to the uncertain flatfield of the C-image at low intensity levels, the colors beyond about $8^{\prime}$ are not reliable. We are particularly reluctant to claim the reality of the outer red structures in the north, but mention that on Schweizer's plate (his Fig. 1) the northern extension of NGC 1316 appears more diffuse than the southern border.

The extended line emission region of Mackie \& Fabbiano (1998) is visible as the bright spot, presumably dust, in the south just above the CCD-gap, which unfortunately covers part of the structure. The southern HII-region, detected by Schweizer (1980), appears as a dark spot not well visible in this display. It is an extremely interesting object, apparently showing globular cluster formation under quite isolated conditions. We intend to dedicate a contribution to this object alone. 


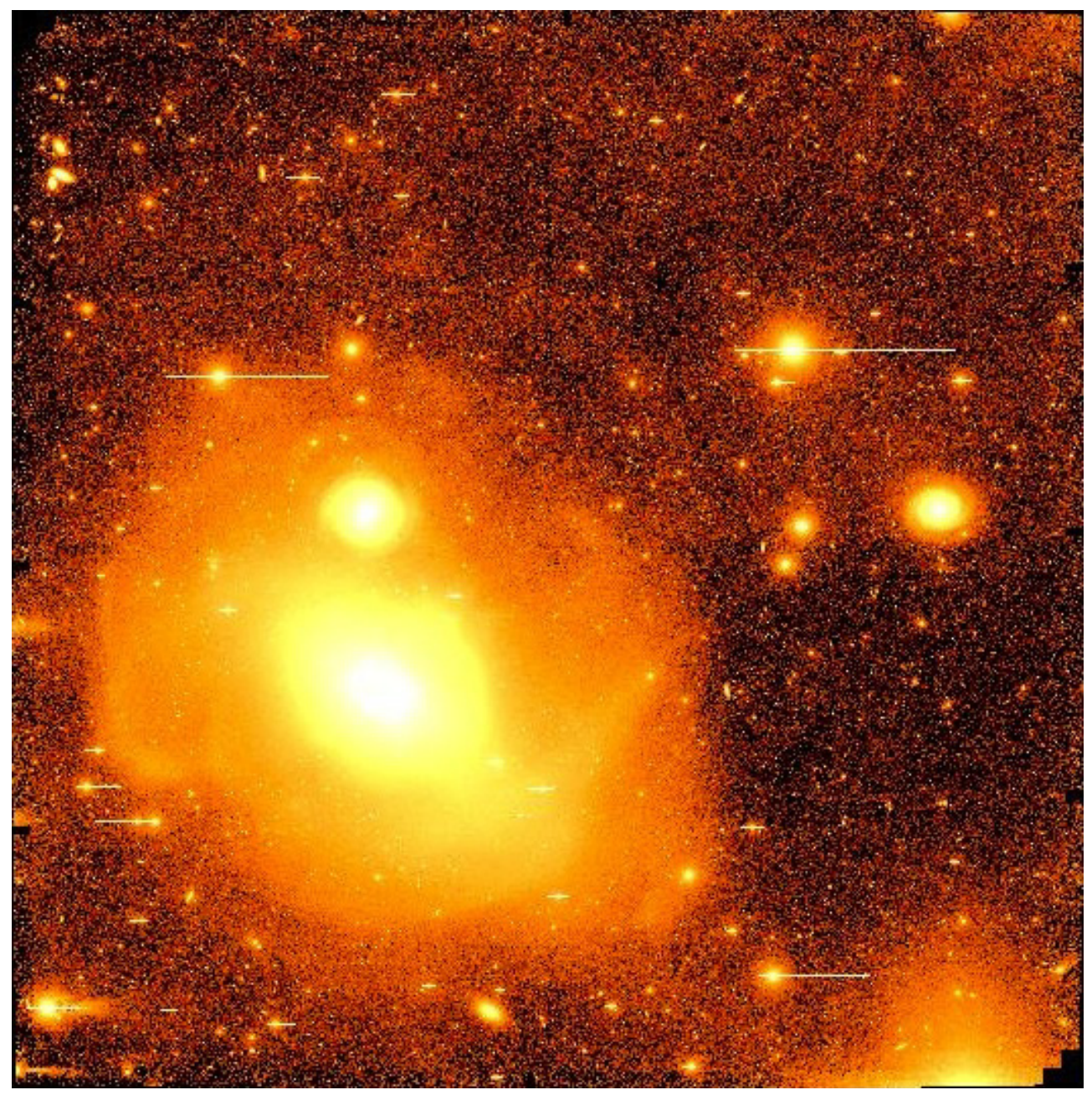

Fig. A.1. Our $R$-image of NGC 1316. The size is $36^{\prime} \times 36^{\prime}$. North is up, east to the left. One notes the relatively well-defined outer border. The diameter along the major axis is $24.5^{\prime}$, along the minor axis $21.5^{\prime}$. The intensity levels are displayed logarithmically. Almost all faint structures are already visible on the photographic image of Schweizer (1980) except for the very faint arc that appears as a continuation of the plume reaching out to the NW. The faint larger-scale structures in the upper part are probably flat-field structures. There is scattered light into the camera at the lower right edge, coming from the $V=7.7$ mag star HD 20914. The border of the globular cluster system corresponds well to the visible luminous body.

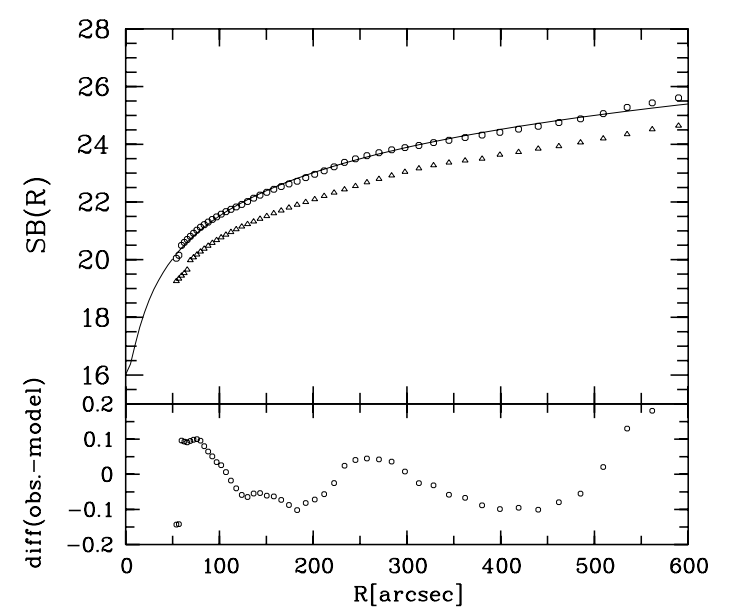

Fig. A.2. Upper panel: our surface-brightness profile in the $R$-band. The solid line is the beta-model. Open circles denote the surface brightness with spherical isophotes. Open triangles denote the surface brightness with elliptical isophotes (ellipticity 0.3 , position angle $50^{\circ}$ ). This profile was shifted by $-0.5 \mathrm{mag}$ for better visibility. Lower panel: residuals in mag between our measurements (upper panel) and the photometric model in the sense: observations-model.

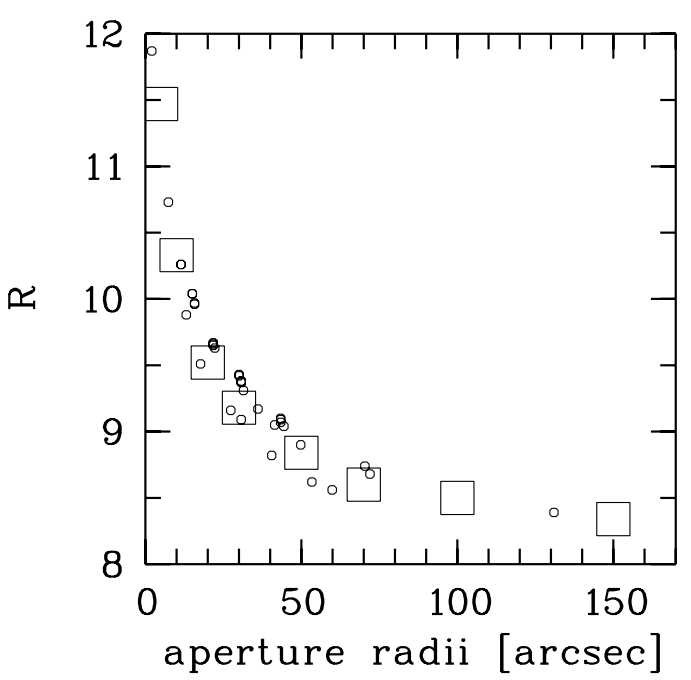

Fig. A.3. Model of our surface-brightness profile reproducing published $R$-band aperture photometries excellently. $x$-axis shows aperture radii, the $y$-axis the apparent $R$ magnitude. Open circles are from the compilation of Prugniel \& Heraudeau (1998), squares are simulated aperture photometries of our beta-model. 


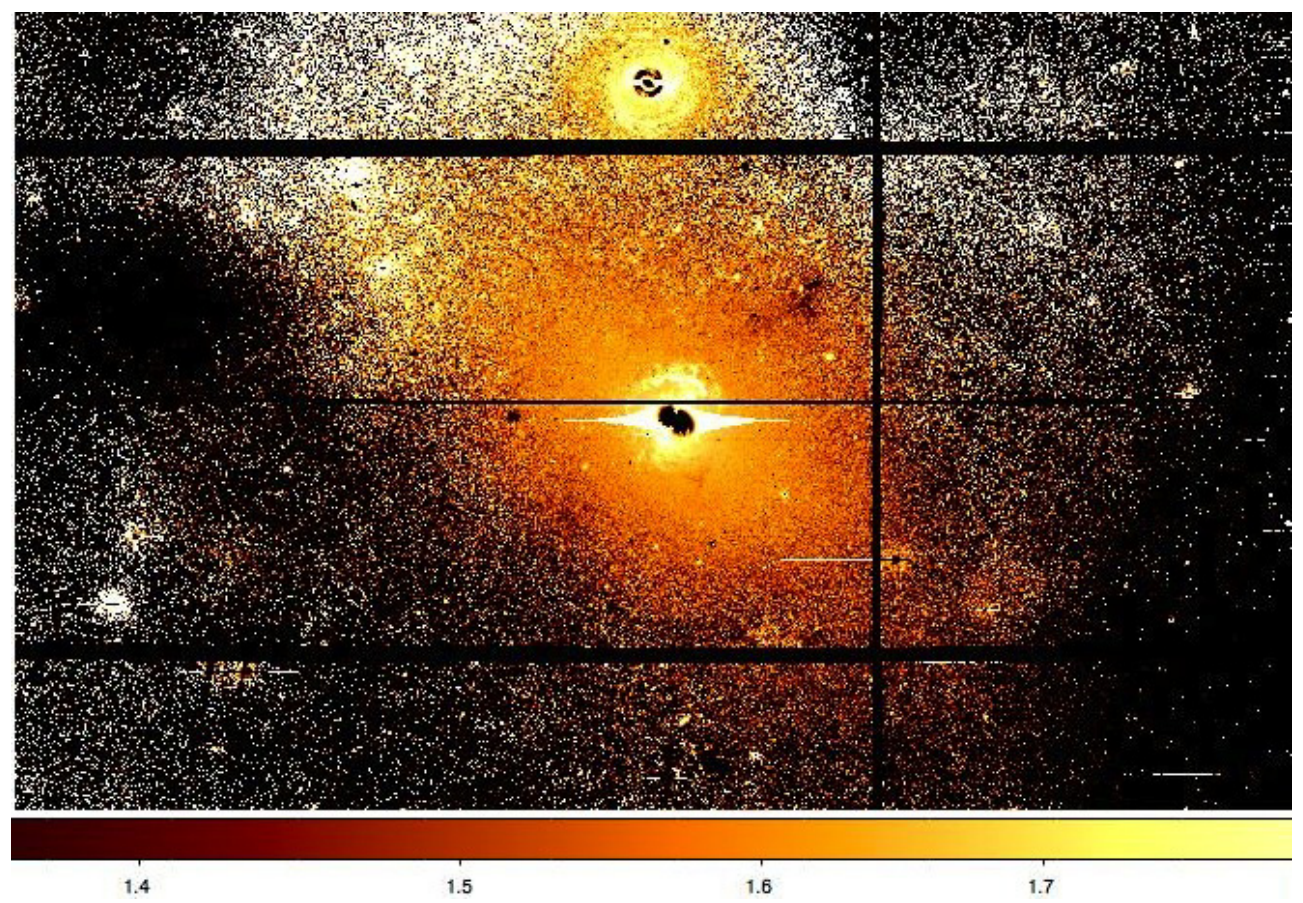

Fig. A.4. Color image of NGC 1316. See text for details.

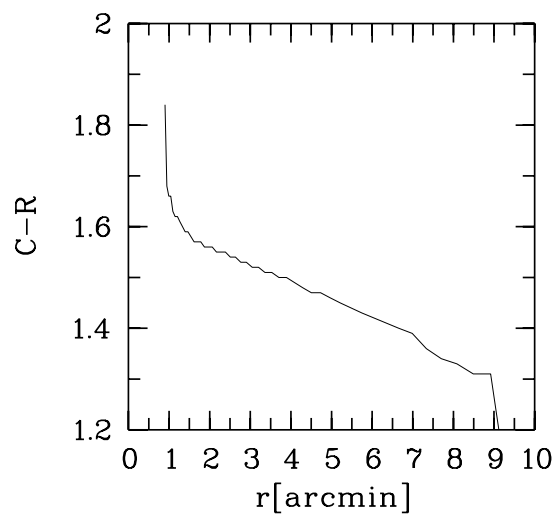

Fig. A.5. Radial color profile. The color was measured along the major axis of NGC 1316 averaging along elliptical isophotes with a position angle of $50^{\circ}$.

\section{A.4. The color profile}

The color map does not exhibit a clear elliptical appearance. Despite this, we adopted the ellipticity of the optical isophotes and evaluated the color by averaging along "color-isophotes" with a fixed ellipticity of 0.3 and a position angle of $50^{\circ}$, corresponding to the major axis of NGC 1316. Figure A.5 shows the radial dependence of color. The inner jump to very red colors is due to the central dust structures. The gradient was noted already by Schweizer (1980), who attributed it to a declining abundance in the outer parts. The question whether age or abundance is the dominant factor, must be left for future investigations of the outer clusters.

\section{A.5. Color image of the central parts}

The wealth of structure and features that is visible in the inner color map deserves a special description. The basis is Fig. A.6.
This map displays $C-R$ in the range from 1.5 to 1.7 . White is red, dark is blue. The reddest colors are caused by dust, while bluer colors are caused by bluer stellar populations and/or perhaps by strong emission of the OII-3727 line, which falls into the $C$-filter. The inner dust features best known from HST images appear white with a color of about $C-R=1.9$. They fit well to nonstellar PAH emission (Temi et al. 2005; Kaneda et al. 2007). Note that the overall symmetry of the dust distribution differs from the spheroid. It shows a slightly elliptical distribution with its major axis at a PA of about $-30^{\circ}$.

Note the many tiny dust patches in the north, which are absent in the south. Remarkable is the slightly curved brighter feature, beginning at about $2.5^{\prime}$ to the north, the PA first being $70^{\circ}$ and $90^{\circ}$ on its eastern part. The overall impression is that of a relic of a spiral arm, which may be a direct clue to the nature of the original galaxy. At its southern border, it is accompanied by a more blueish color, recalling that dust usually follows trailing spiral arms.

One of the most conspicuous features is Schweizer's "plume", pointing toward the NW. Neither Schweizer nor Mackie \& Fabbiano (1998) detected line emission. No atomic or molecular gas has been detected (Horellou et al. 2001). Dust also seems to be absent. Its total extent in the NW-direction is almost $2^{\prime}$, corresponding to $10 \mathrm{kpc}$. One finds the bluest color at the "head" in some blue spots which have $C-R=1.1$. Toward the SE, the color becomes somewhat redder, typically 1.4 . Since we observe in projection, this does not mean that the color of the associated stellar population is really varying, but only that its density varies. The true color might be quite blue and homogenous. Unless this population has a low metallicity (an infalling dwarf galaxy in tidal dissolution cannot be excluded), it must be younger than 1 Gyr. Graham (1987) made the interesting suggestion that the plume might represent young stars whose formation was triggered by a nuclear jet. A correspondence might be seen in a group of blue stars in CenA that has its probable origin in star formation triggered by the X-ray jet (Graham $\&$ Fassett 2002). However, its major axis points neither to the 


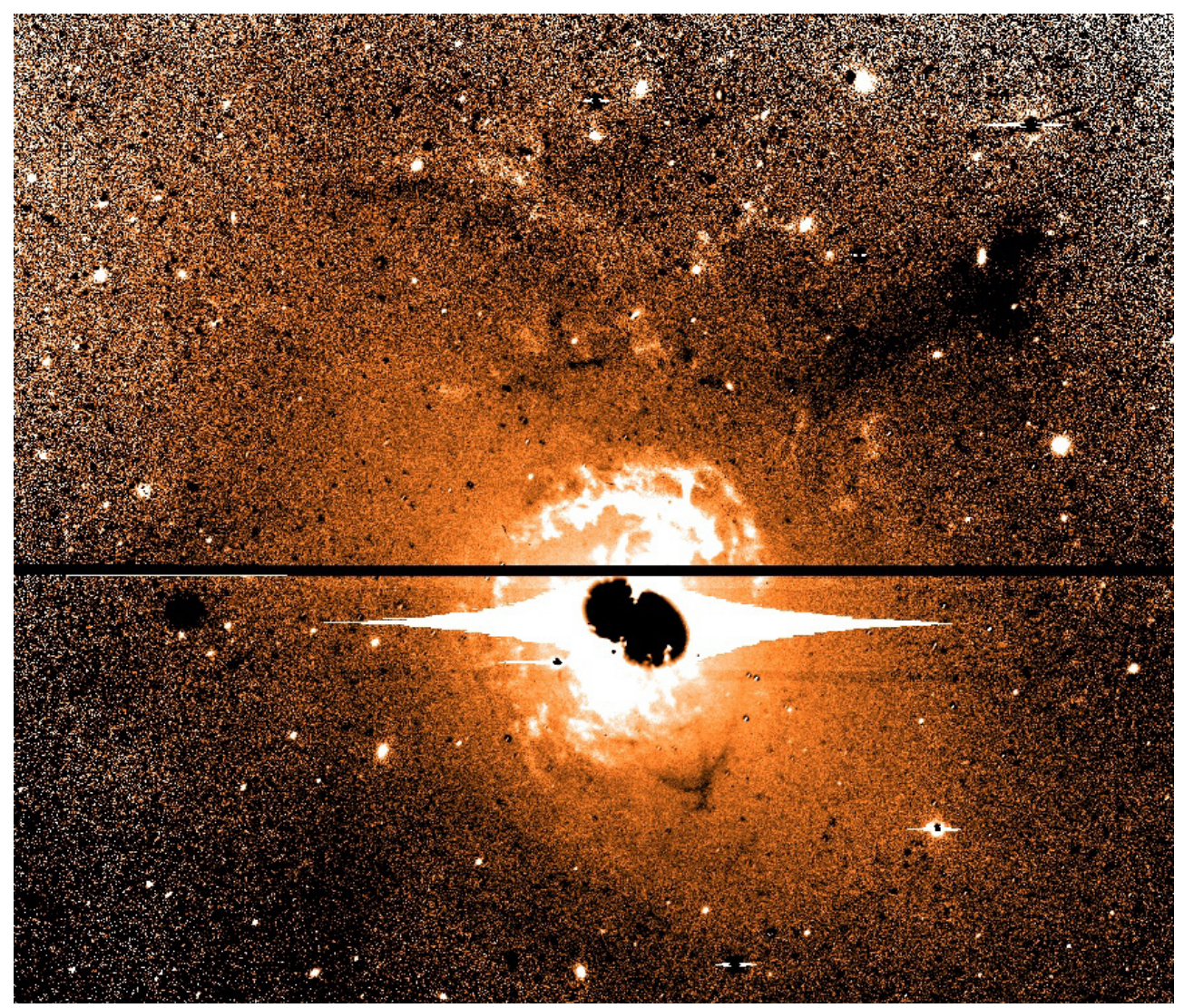

Fig. A.6. Inner part of NGC 1316 showing the $C-R$ color map. The size is $7.8^{\prime} \times 6.5^{\prime}$. Red is bright, blue is dark. The dynamical range is $1.5<C-R<1.7$, so also tiny color differences become visible. See text for details.

nucleus nor to the center of the western inner radio lobe (which is not necessarily a counterargument after roughly a crossing time). The alternative (also mentioned by Graham 1987) is an infalling dwarf galaxy in dissolution, which appears to be more probable.
Striking is also the psi-shaped irregular structure $1^{\prime}$ to the south, which is bluer than its environment by $0.05 \mathrm{mag}$, which to our knowledge has not yet been mentioned in the literature. Whether the color is caused by a bluer population or due to line emission, must be cleared up by spectroscopy. 